Supporting information for:

\title{
NMR Quantification of the Effects of Ligands and Counterions on Lewis Acid Catalysis
}

\author{
Julia J. Jennings, Ben W. Wigman, Brittany M. Armstrong, Annaliese K. Franz* \\ Department of Chemistry, One Shields Ave, University of California, Davis, CA 95616 USA
}

Supporting Information

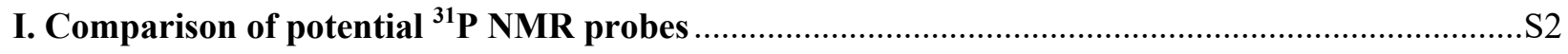

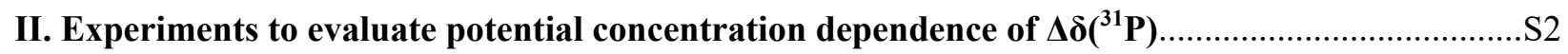

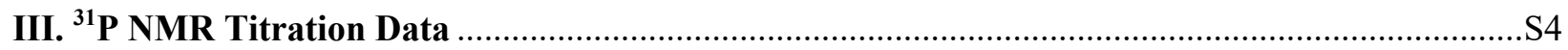

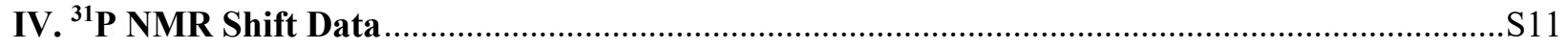

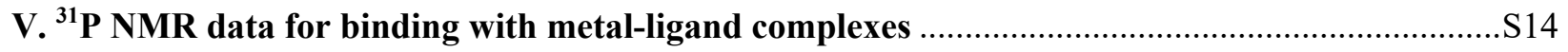

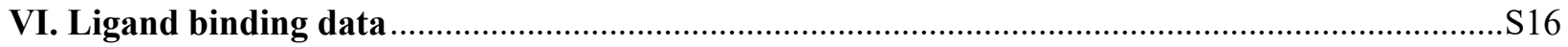

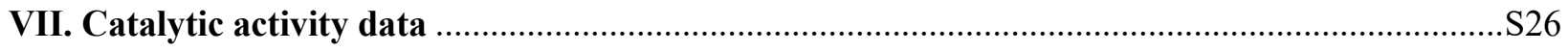

VIII. Correlation of $\boldsymbol{\Delta} \delta\left({ }^{31} \mathrm{P}\right)$, charge density and catalytic activity .............................................. 331 


\section{Comparison of potential ${ }^{31} \mathrm{P}$ NMR probes}

Table S1. Comparison of ${ }^{31} \mathrm{P}$ NMR probes

\begin{tabular}{|c|c|c|c|c|}
\hline & $\begin{array}{c}\text { O } \\
\text { Et }-\mathbf{P}_{\text {Et }}^{\text {I }}-\mathbf{E t} \\
\text { Triethylphosphine } \\
\text { Oxide } \\
\text { (TEPO) }\end{array}$ & $\begin{array}{c}\text { M } \\
\begin{array}{c}\text { M } \\
\text { Eto-P } \\
\text { Eto }\end{array} \\
\text { tetraethyl } \\
\text { methylenebis } \\
\text { (phosphonate) } \\
\text { (TEMBP) }\end{array}$ & 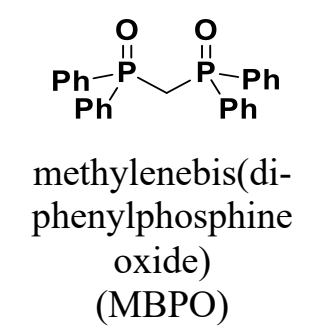 & 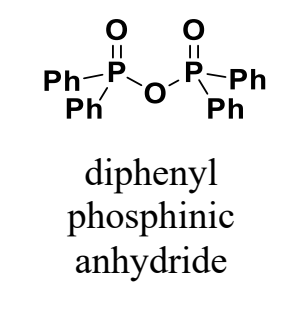 \\
\hline $\begin{array}{l}\text { Commercially } \\
\text { available? }\end{array}$ & Yes & Yes & $\mathrm{No}^{5}$ & Yes \\
\hline $\begin{array}{l}\text { Maximum } \\
\Delta \delta\left({ }^{31} \mathrm{P}\right) \\
\text { observed }\end{array}$ & $50 \mathrm{ppm}$ & $4.0 \mathrm{ppm}$ & $\begin{array}{l}30 \mathrm{ppm}\left({ }^{31} \mathrm{P} \text { NMR }\right) \\
1.2 \mathrm{ppm}\left({ }^{1} \mathrm{H} \text { NMR }\right)\end{array}$ & $16+\mathrm{ppm}$ \\
\hline $\begin{array}{l}\text { Other } \\
\text { considerations }\end{array}$ & & & $\begin{array}{l}\text { probe can also be } \\
\text { used to provide } \\
{ }^{1} \mathrm{H} \text { NMR data }\end{array}$ & $\begin{array}{l}\text { anhydride may be } \\
\text { unstable, spectra } \\
\text { often show } \\
\text { several peaks }\end{array}$ \\
\hline $\begin{array}{l}\delta\left({ }^{31} \mathrm{P}\right) \mathrm{NMR} \text { in } \\
\mathrm{CD}_{2} \mathrm{Cl}_{2}(\mathrm{ppm})\end{array}$ & 50.3 & 19.1 & $\begin{array}{l}23.8\left({ }^{31} \mathrm{P} \text { NMR }\right) \\
3.52\left({ }^{1} \mathrm{H} \text { NMR }\right)\end{array}$ & \\
\hline
\end{tabular}

\section{Experiments to evaluate potential concentration dependence of $\Delta \delta\left({ }^{31} \mathrm{P}\right)$}

Binding equilibrium is potentially affected by concentration. Two concentrations were used throughout the study. Binding studies summarized in Table 1 were performed at $16 \mathrm{mM}$ with respect to the Lewis base and $48 \mathrm{mM}$ of the Lewis acid, while binding studies summarized in Table 2 were performed at 4.0 $\mathrm{mM}$ with respect to the Lewis base and $12 \mathrm{mM}$ of the Lewis acid. The higher concentration (48 $\mathrm{mM}$ Lewis acid) studies resulted in stronger ${ }^{31} \mathrm{P}$ NMR signal and require fewer scans and therefore less instrument time to observe clear spectra. The lower concentration (12 mM Lewis acid) more closely matches reaction conditions (assuming a $0.1 \mathrm{M}$ reaction with $10 \mathrm{~mol} \%$ catalyst loading) and maximizes the amount of Lewis acid, ligand and additive dissolved. To evaluate how this difference in concentration would affect the ability to compare data between binding study concentrations, the concentration dependence of $\Delta \delta\left({ }^{31} \mathrm{P}\right)$ data was evaluated for two common Lewis acids: $\mathrm{TiCl}_{4}$, a fully soluble Lewis acid that can be easily added as a solution, and $\mathrm{Sc}(\mathrm{OTf})_{3}$, a Lewis acid salt that is sparingly soluble in $\mathrm{CH}_{2} \mathrm{Cl}_{2}$. 
Although $\mathrm{Sc}(\mathrm{OTf})_{3}$ and similar metal salts are only sparingly soluble in organic solvents such as $\mathrm{CH}_{2} \mathrm{Cl}_{2}$ and toluene, these Lewis acids have well documented catalytic activity in these solvents.

\section{Procedure for TEPO-binding concentration experiments}

Into an oven-dried, Ar-purged NMR tube fitted with a septa cap was added the necessary amount of Lewis acid (as a solid or as $1.0 \mathrm{M}$ in $\mathrm{CH}_{2} \mathrm{Cl}_{2}$ ) and TEPO (from a prepared $0.2 \mathrm{M}$ stock solution in $\mathrm{CH}_{2} \mathrm{Cl}_{2}$ ) to give the desired concentration at a 1:1 molar ratio, followed by $0.1 \mathrm{~mL}$ of $\mathrm{CD}_{2} \mathrm{Cl}_{2}$ and the required volume of $\mathrm{CH}_{2} \mathrm{Cl}_{2}$ to give a final volume of $0.5 \mathrm{~mL}$. The ${ }^{31} \mathrm{P}$ NMR spectrum of each sample was collected at room temperature with 128 scans and compared to a standard of TEPO in $\mathrm{CD}_{2} \mathrm{Cl}_{2}$.

\section{$\Delta \delta\left({ }^{31} \mathrm{P}\right)$ Data for $\mathrm{Sc}(\mathrm{OTf})_{3}$ binding to TEPO do not vary with concentration from 4.0 to $32 \mathrm{mM}$}

General binding experiments were conducted at $16 \mathrm{mM}$ of ${ }^{31} \mathrm{P}$ NMR probe (Table 1). To maximize solubility of metal-ligand complexes in organic solvents such as $\mathrm{CH}_{2} \mathrm{Cl}_{2}$ and toluene, binding experiments shown in Table 2 were conducted at $4.0 \mathrm{mM}$. Due to this difference in concentration, binding studies were performed at varying concentration to determine the effect of concentration on $\Delta \delta\left({ }^{31} \mathrm{P}\right)$ data. The observed $\Delta \delta\left({ }^{31} \mathrm{P}\right)$ data for TEPO binding with $\mathrm{Sc}(\mathrm{OTf})_{3}$ remained constant from 4.0 to $32 \mathrm{mM}$ (Figure $\mathrm{S} 1$ ).

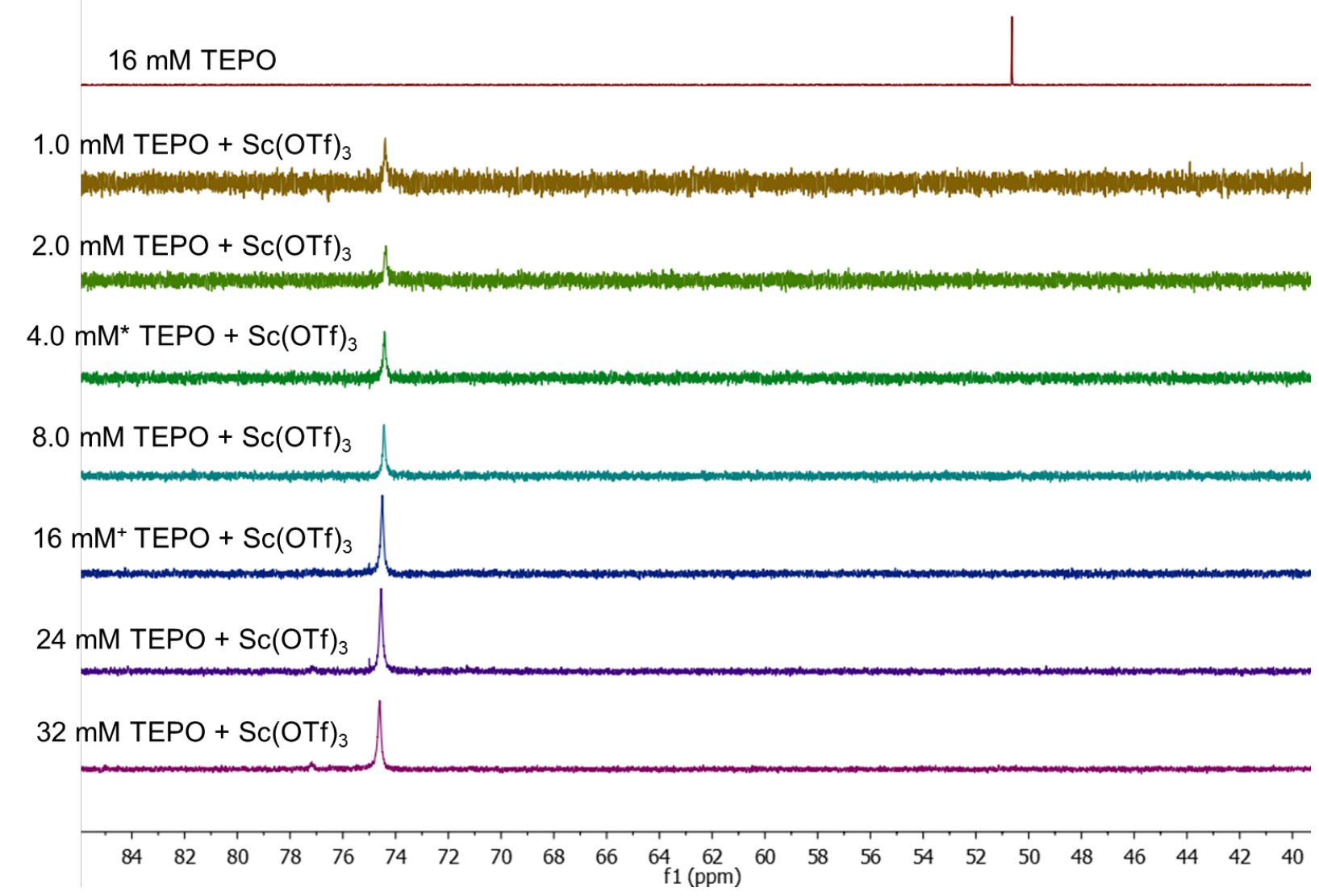


Figure S1. Effect of concentration on $\Delta \delta\left({ }^{31} \mathrm{P}\right)$ data for TEPO as $1: 1$ mixtures of $\mathrm{Sc}(\mathrm{OTf})_{3}$ and TEPO in 4:1 $\mathrm{CH}_{2} \mathrm{Cl}_{2} / \mathrm{CD}_{2} \mathrm{Cl}_{2}$. ${ }^{+}$Concentration used in general binding studies reported in Table 1. ${ }^{*}$ Concentration used in metal-ligand binding studies (Table 2).

\section{$\Delta \delta\left({ }^{31} \mathrm{P}\right)$ Data for $\mathrm{TiCl}_{4}$ binding to TEPO vary with concentration}

The observed $\Delta \delta\left({ }^{31} \mathrm{P}\right)$ data for TEPO binding with $\mathrm{TiCl}_{4}$ increases with increasing concentration from 4.0 $\mathrm{mM}$ to $64 \mathrm{mM}$ (Figure S2). Binding experiments were performed at $16 \mathrm{mM}$ final concentration of the phosphorous probe for Table 1.

$16 \mathrm{mM}$ TEPO

4.0 $\mathrm{mM}$ TEPO $+\mathrm{TiCl}_{4}$

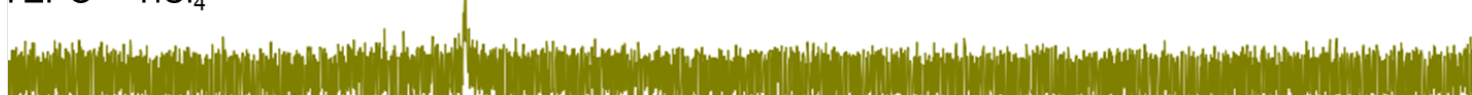

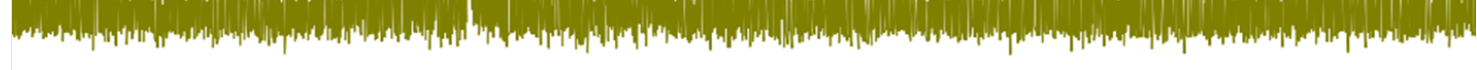

$8.0 \mathrm{mM}$ TEPO + $\mathrm{TiCl}_{4}$

$16 \mathrm{mM}^{*} \mathrm{TEPO}+\mathrm{TiCl}_{4}$

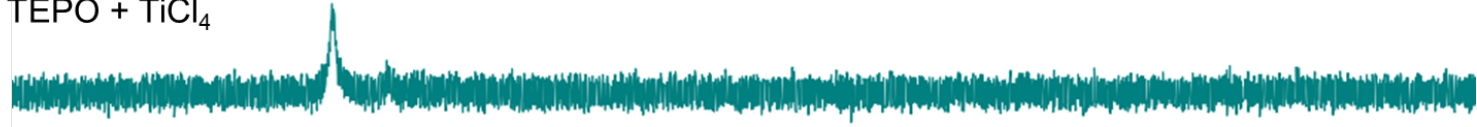

$32 \mathrm{mM}$ TEPO $+\mathrm{TiCl}_{4}$

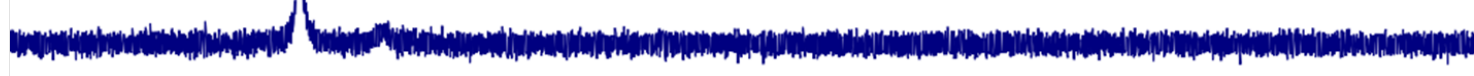

$64 \mathrm{mM}$ TEPO $+\mathrm{TiCl}_{4}$

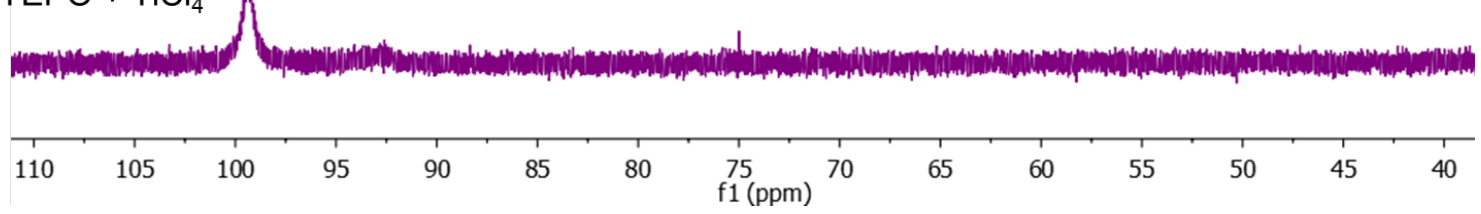

Figure S2. Effect of concentration on ${ }^{31} \mathrm{P}$ NMR shift for TEPO as 1:1 mixtures of $\mathrm{TiCl}_{4}$ and TEPO in 4:1

$\mathrm{CH}_{2} \mathrm{Cl}_{2} / \mathrm{CD}_{2} \mathrm{Cl}_{2}$. ${ }^{*}$ Concentration used in binding studies reported in Table 1 .

\section{III. ${ }^{31}$ P NMR Titration Data}

To determine the stoichiometry of ${ }^{31} \mathrm{P}$ NMR probe binding to Lewis acid, we investigated $\mathrm{TiCl}_{4}$ binding to TEPO as an idealized representative example due to its solubility. $\mathrm{Sc}(\mathrm{OTf})_{3}$ was investigated as a representative for less soluble but catalytically active and important Lewis acids. TEPO concentration was held constant at $16 \mathrm{mM}$ for titration experiments and varying amounts of Lewis acid were added to give 
the desired equivalents. Due to the presence of multiple peaks in the observed ${ }^{31} \mathrm{P}$ NMR spectra, continuous variation was not observed, and Job plot analysis could not be performed.

IIIa. Experiments to determine TEPO binding stoichiometry with Lewis acids

TEPO standard

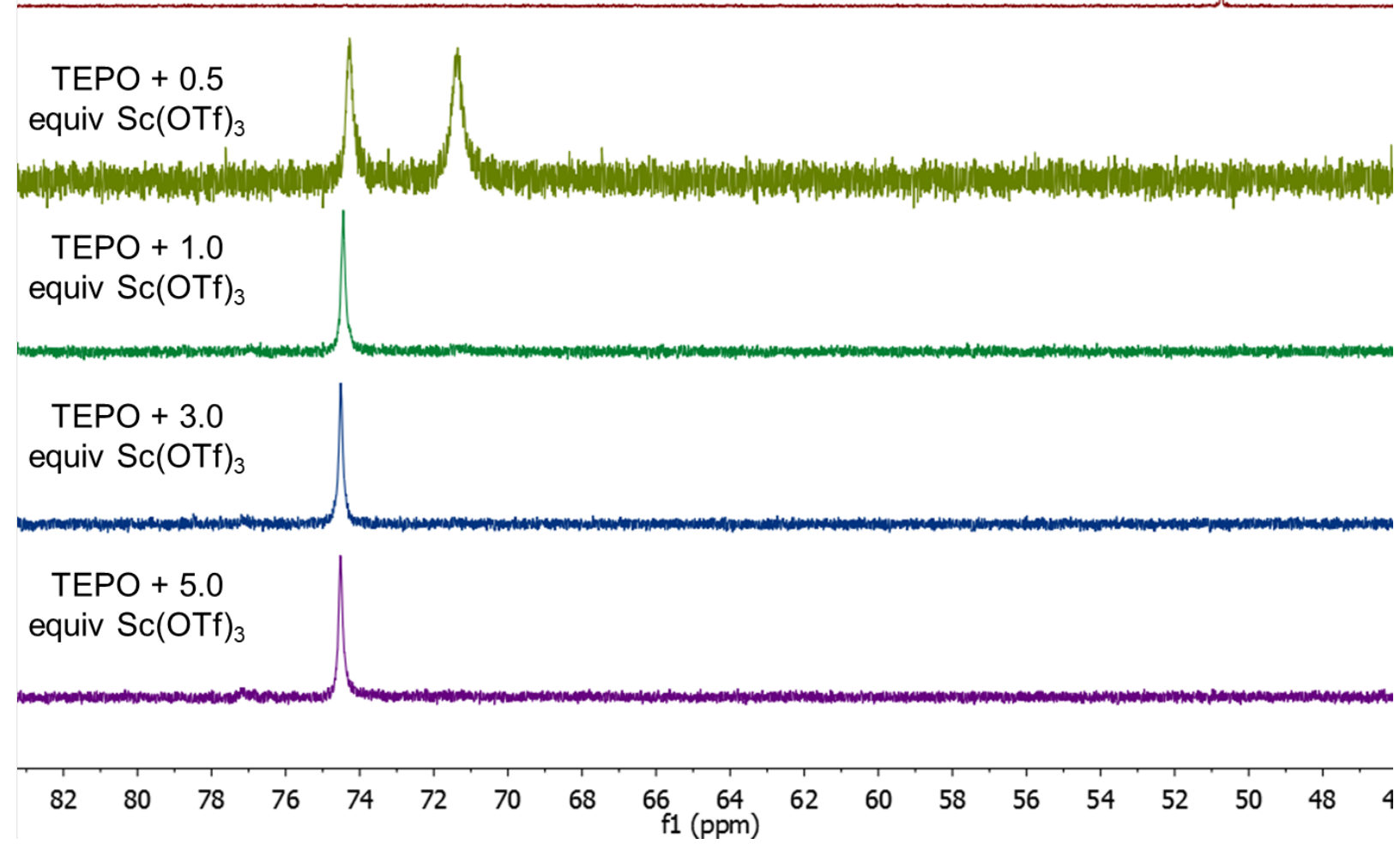

Figure S3. Effect of molar ratio on $\Delta \delta\left({ }^{31} \mathrm{P}\right)$ data for $\mathrm{TEPO} / \mathrm{Sc}(\mathrm{OTf})_{3}$ mixtures.

Table S2. ${ }^{31} \mathrm{P}$ NMR data for titration experiment with $\mathrm{Sc}(\mathrm{OTf})_{3}$

\begin{tabular}{ccc}
\hline Equiv of $\mathrm{Sc}(\mathrm{OTf})_{3}$ & $\delta\left({ }^{31} \mathrm{P}\right)(\mathrm{ppm})$ & $\Delta \delta\left({ }^{31} \mathrm{P}\right)(\mathrm{ppm})$ \\
\hline 0 & 50.3 & 0.0 \\
0.5 & $73.8,70.9^{\mathrm{a}}$ & $23.5,20.6^{\mathrm{a}}$ \\
1.0 & 74.0 & 23.7 \\
3.0 & 74.1 & 23.8 \\
5.0 & 74.1 & 23.8
\end{tabular}


${ }^{a}$ Multiple peaks observed with less than one equivalent of Lewis acid to TEPO could be attributed to multiple binding modes e.g. more than one TEPO molecule binding to $\mathrm{Sc}(\mathrm{OTf})_{3}$ as well as a $1: 1$ $\mathrm{TEPO} / \mathrm{Sc}(\mathrm{OTf})_{3}$ in solution.

\section{TEPO standard}

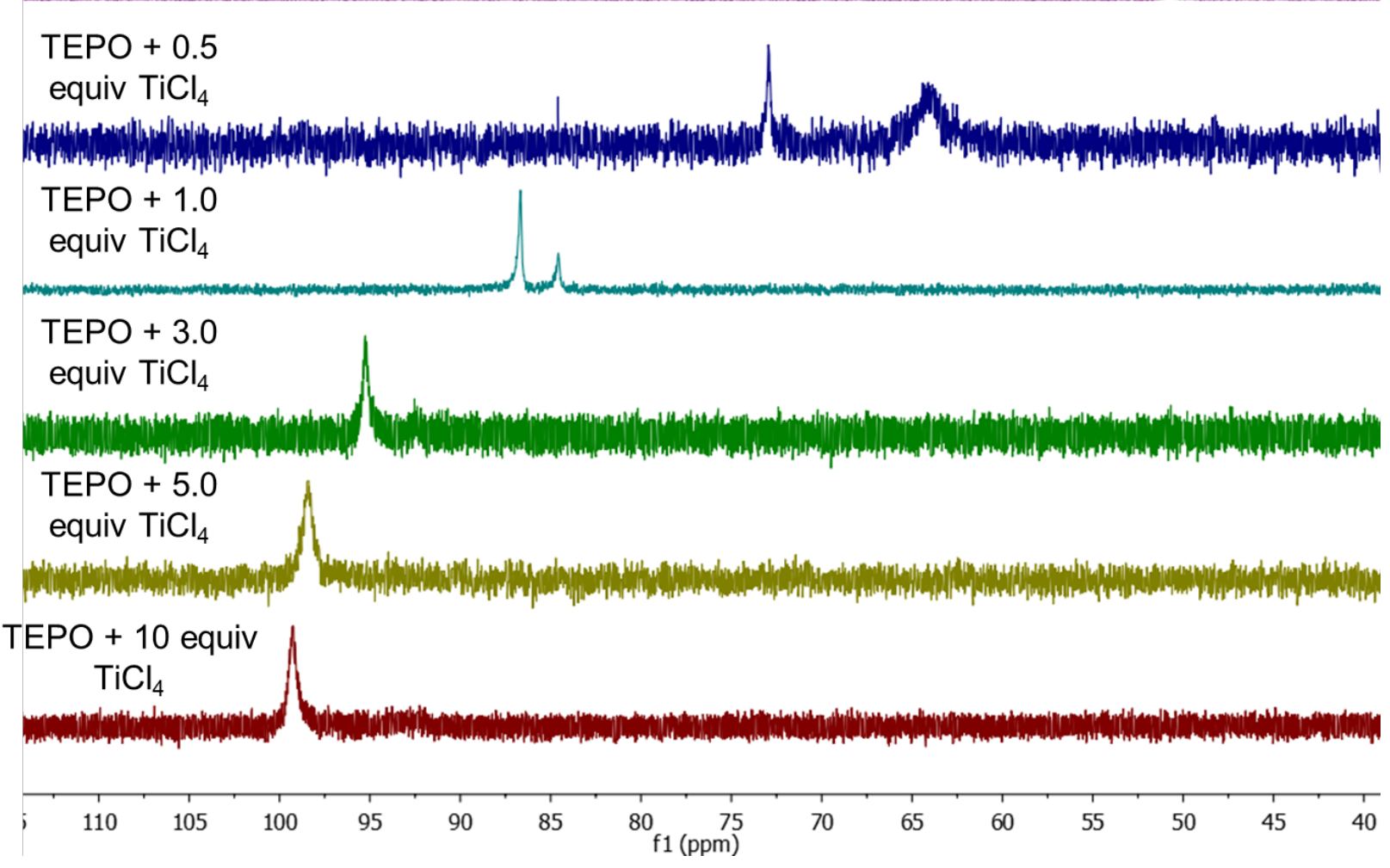

Figure S4. Effect of molar ratio on $\Delta \delta\left({ }^{31} \mathrm{P}\right)$ data for $\mathrm{TEPO} / \mathrm{TiCl}_{4}$ mixtures.

Table S3. ${ }^{31} \mathrm{P}$ NMR data for titration experiment with $\mathrm{TiCl}_{4}$

\begin{tabular}{ccc}
\hline Equiv of $\mathrm{TiCl}_{4}$ & $\delta\left({ }^{31} \mathrm{P}\right)(\mathrm{ppm})$ & $\Delta \delta\left({ }^{31} \mathrm{P}\right)(\mathrm{ppm})$ \\
\hline 0 & 50.3 & 0 \\
0.5 & $72.6,63.8^{\mathrm{a}}$ & $22.3,13.5^{\mathrm{a}}$ \\
1.0 & $86.4,84.3^{\mathrm{a}}$ & $36.1,34.0^{\mathrm{a}}$ \\
3.0 & 94.9 & 44.6 \\
5.0 & 98.1 & 47.8 \\
10 & 98.9 & 48.6
\end{tabular}

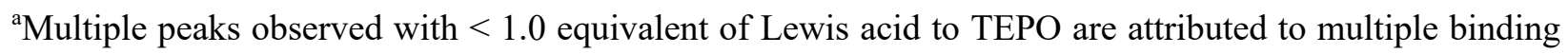
modes, e.g. 2:1 TEPO/TiCl 4 as well as 1:1 TEPO/TiCl ${ }_{4}$ in solution 
TEPO standard

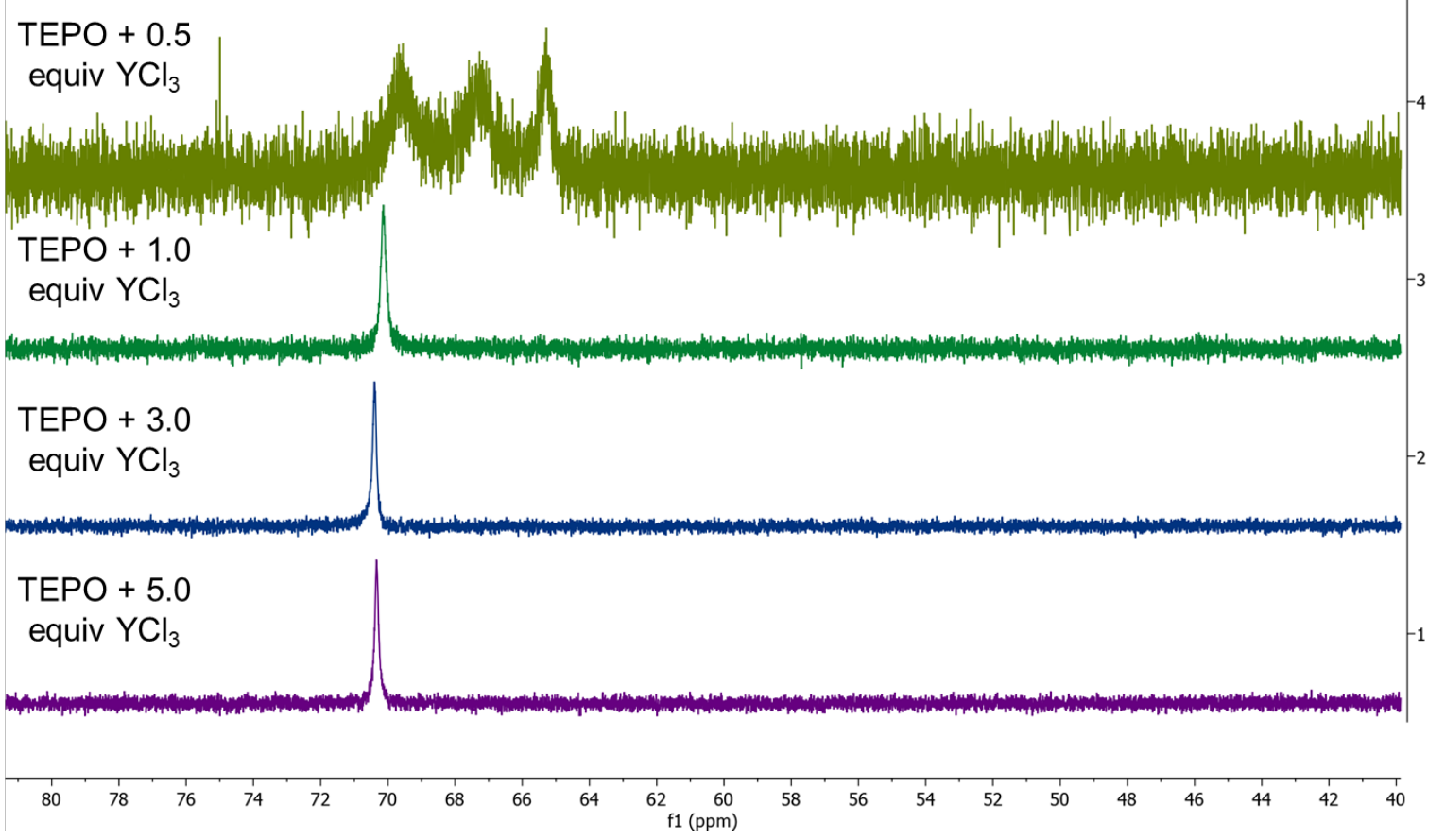

Figure S5. Effect of molar ratio on $\Delta \delta\left({ }^{31} \mathrm{P}\right)$ data for $\mathrm{TEPO} / \mathrm{YCl}_{3}$ mixtures.

Table S4. ${ }^{31} \mathrm{P}$ NMR data for titration experiment with $\mathrm{YCl}_{3}$

\begin{tabular}{ccc}
\hline Equiv of $\mathrm{YCl}_{3}$ & $\delta\left({ }^{31} \mathrm{P}\right)(\mathrm{ppm})$ & $\Delta \delta\left({ }^{31} \mathrm{P}\right)(\mathrm{ppm})$ \\
\hline 0 & 50.3 & 0 \\
0.5 & $69.3,66.9,64.9^{\mathrm{a}}$ & $19.0,16.6,14.6^{\mathrm{a}}$ \\
1.0 & 69.7 & 19.4 \\
3.0 & 70.0 & 19.7 \\
5.0 & 69.9 & 19.6
\end{tabular}

${ }^{a}$ Multiple peaks observed with less than one equivalent of Lewis acid to TEPO are attributed to multiple binding modes e.g. 2:1 TEPO/YCl 3 as well as 1:1 TEPO/YCl $\mathrm{Yin}_{3}$ solution. 


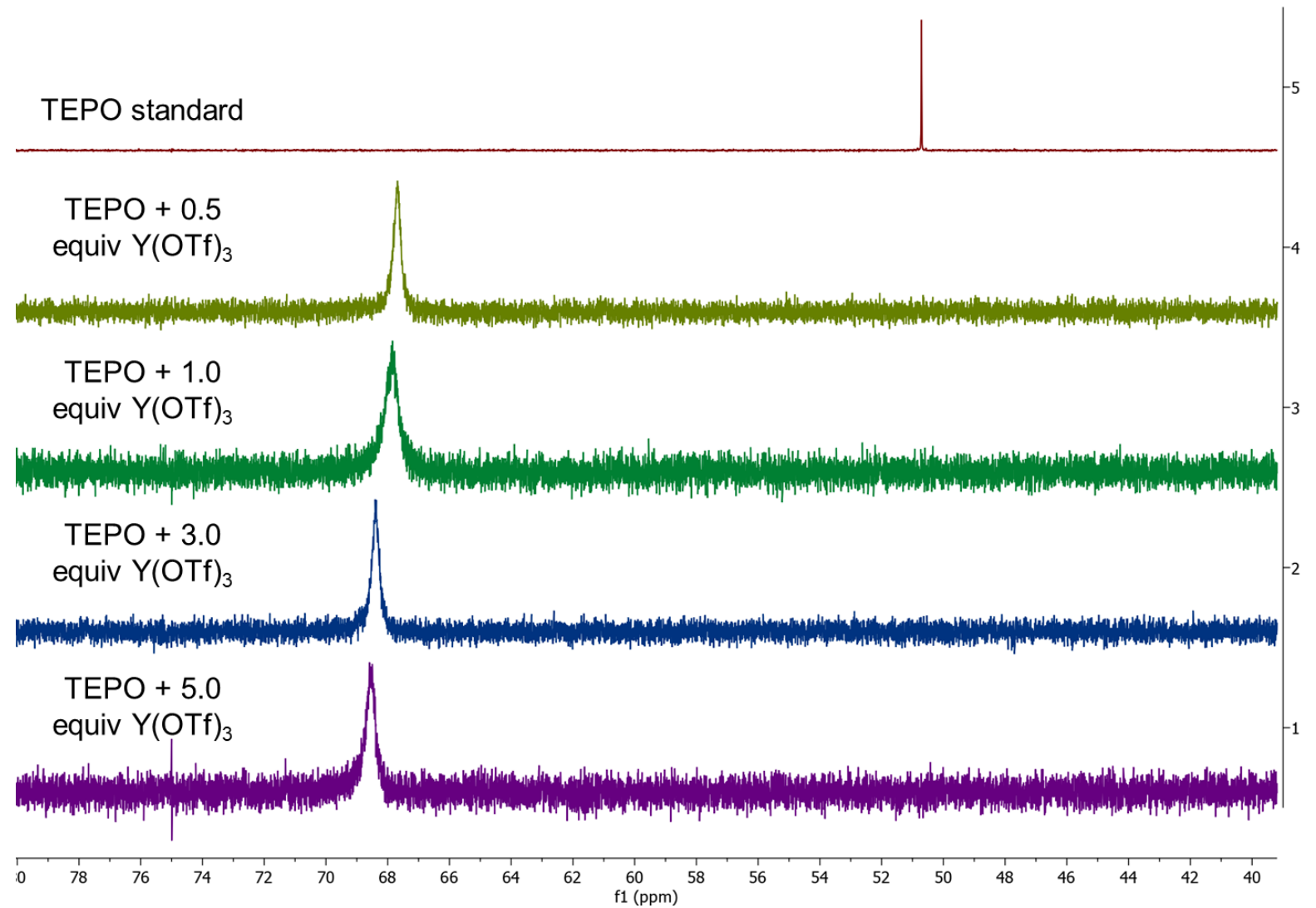

Figure S6. Effect of molar ratio on $\Delta \delta\left({ }^{31} \mathrm{P}\right)$ data for TEPO/Y $(\mathrm{OTf})_{3}$ mixtures.

Table S5. ${ }^{31} \mathrm{P}$ NMR data for titration experiment with $\mathrm{Y}(\mathrm{OTf})_{3}$

\begin{tabular}{ccc}
\hline Equiv of $\mathrm{Y}(\mathrm{OTf})_{3}$ & $\delta\left({ }^{31} \mathrm{P}\right)(\mathrm{ppm})$ & $\Delta \delta\left({ }^{31} \mathrm{P}\right)(\mathrm{ppm})$ \\
\hline 0 & 50.3 & 0 \\
0.5 & 67.3 & 17.0 \\
1.0 & 67.4 & 17.1 \\
3.0 & 68.0 & 17.7 \\
5.0 & 68.1 & 17.8
\end{tabular}


IIIb. Experiments to determine TEMBP binding stoichiometry with Lewis acids

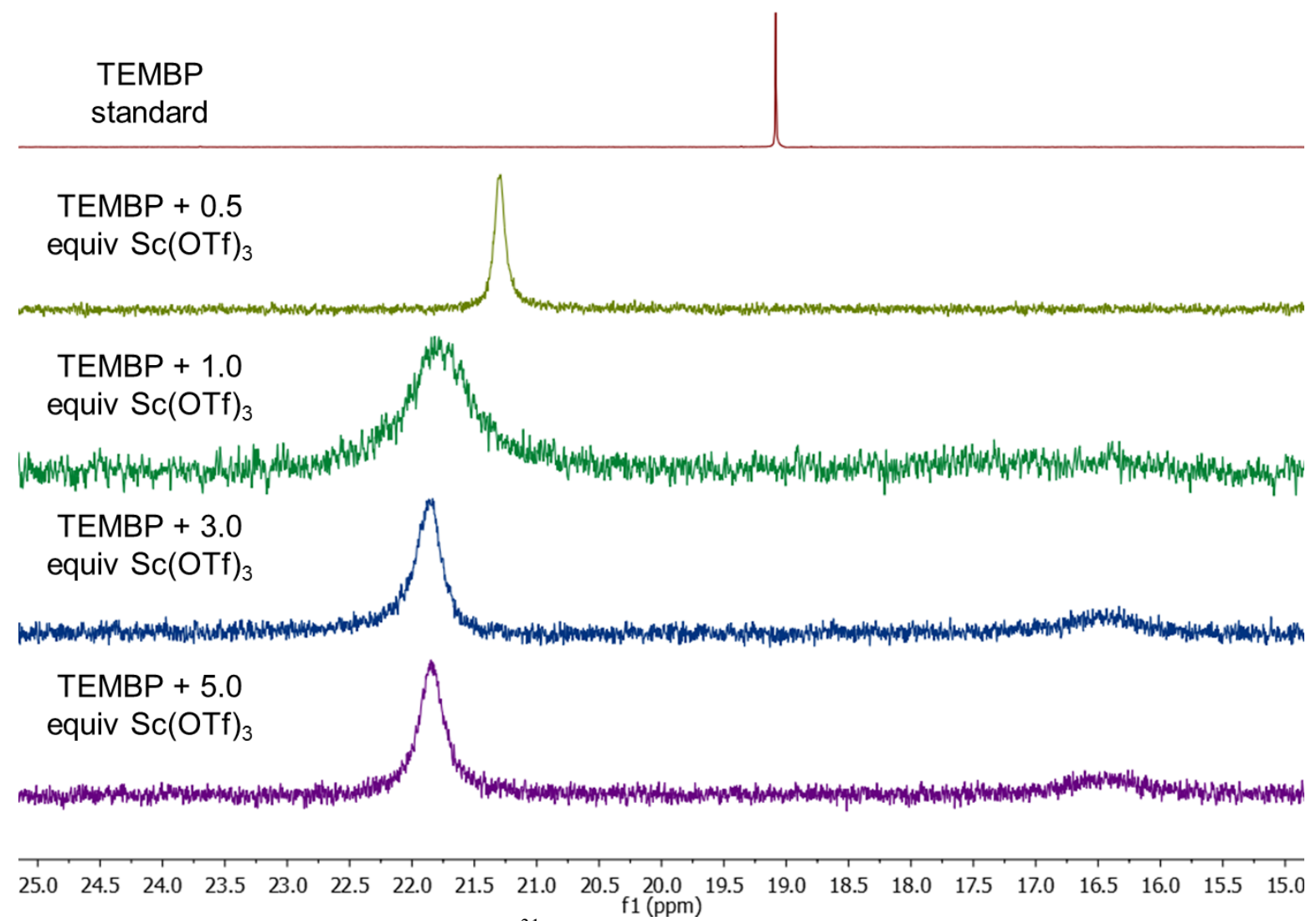

Figure S7. Effect of molar ratio on $\Delta \delta\left({ }^{31} \mathrm{P}\right)$ data for $\mathrm{TEPO} / \mathrm{Sc}(\mathrm{OTf})_{3}$ mixtures.

Table S6. ${ }^{31} \mathrm{P}$ NMR spectroscopy data for titration experiment with $\mathrm{Sc}(\mathrm{OTf})_{3}$

\begin{tabular}{ccc}
\hline Equivalents of $\mathrm{Sc}(\mathrm{OTf})_{3}$ & $\delta\left({ }^{31} \mathrm{P}\right)(\mathrm{ppm})$ & $\Delta \delta\left({ }^{31} \mathrm{P}\right)(\mathrm{ppm})$ \\
\hline 0 & 19.1 & 0 \\
0.5 & $21.3^{\mathrm{a}}$ & 2.2 \\
1.0 & $21.8^{\mathrm{b}}$ & 2.7 \\
3.0 & $21.9^{\mathrm{b}, \mathrm{c}}$ & 2.8 \\
5.0 & $21.9^{\mathrm{b}, \mathrm{c}}$ & 2.8
\end{tabular}

${ }^{\text {a}}$ Peak attributed to $1:<1 \quad \mathrm{TEMBP} / \mathrm{Sc}(\mathrm{OTf})_{3}$ binding stoichiometry. ${ }^{\mathrm{b}} \mathrm{Peak}$ attributed to $1: 1$ $\mathrm{TEMBP} / \mathrm{Sc}(\mathrm{OTf})_{3}$ binding stoichiometry. ${ }^{\mathrm{c}} \mathrm{An}$ additional peak at $\Delta \delta\left({ }^{31} \mathrm{P}\right)=16.4 \mathrm{ppm}, \Delta \delta\left({ }^{31} \mathrm{P}\right)=-2.6 \mathrm{ppm}$ was observed. This peak attributed to $1:>1 \mathrm{TEMBP} / \mathrm{Sc}(\mathrm{OTf})_{3}$ binding stoichiometry. 


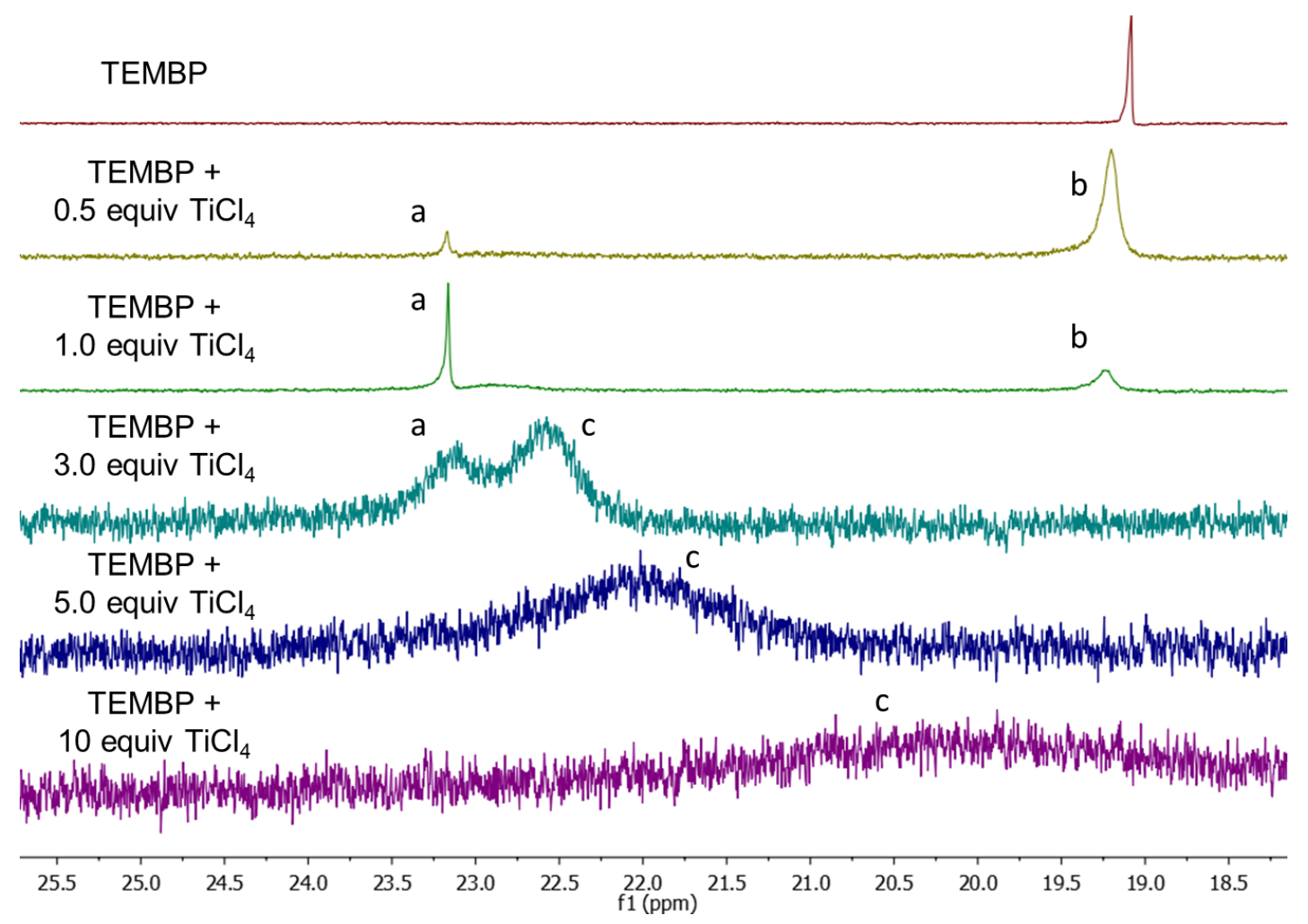

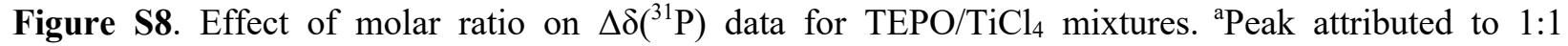
TEMBP/TiCl 4 binding stoichiometry. ${ }^{b}$ Peak attributed to unbound TEMBP. ${ }^{\mathrm{c}}$ Peak attributed to 1:>1 $\mathrm{TEMBP} / \mathrm{TiCl}_{4}$ binding stoichiometry.

Table S7. ${ }^{31} \mathrm{P}$ NMR data for titration experiment with $\mathrm{TiCl}_{4}$

\begin{tabular}{ccc}
\hline Equiv of $\mathrm{TiCl}_{4}$ & $\delta\left({ }^{31} \mathrm{P}\right)(\mathrm{ppm})$ & $\Delta \delta\left({ }^{31} \mathrm{P}\right)(\mathrm{ppm})$ \\
\hline 0 & 19.1 & 0 \\
0.5 & $23.2,{ }^{\mathrm{a}} 19.2^{\mathrm{b}}$ & $4.1,0.1$ \\
1 & $23.2,{ }^{\mathrm{a}} 19.2^{\mathrm{b}}$ & $4.1,0.1$ \\
3 & $23.1,{ }^{\mathrm{a}} 22.6^{\mathrm{c}}$ & $4.0,3.5$ \\
5 & $22.0^{\mathrm{c}}$ & 2.9 \\
10 & $20.1^{\mathrm{c}}$ & 1.0
\end{tabular}

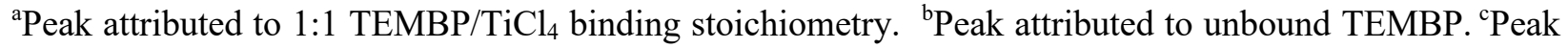
attributed to $1:>1 \mathrm{TEMBP} / \mathrm{TiCl}_{4}$ binding stoichiometry. 


\section{IV. ${ }^{31}$ P NMR Shift Data}

\section{IVa. ${ }^{31}$ P NMR data for TEPO binding}

Select catalysts were run in triplicate to check the reproducibility of the method. Standard error of the mean ranged from 0 to $1.6 \mathrm{ppm}$.

Table S8. $\Delta \delta\left({ }^{31} \mathrm{P}\right)$ NMR measurements for TEPO binding to Lewis acids.

\begin{tabular}{|c|c|c|c|c|c|c|}
\hline Lewis acid & $\begin{array}{c}\text { Trial 1 } \\
\Delta \delta\left({ }^{31} \mathrm{P}\right) \\
(\mathrm{ppm}) \\
\end{array}$ & $\begin{array}{c}\text { Trial 2 } \\
\Delta \delta\left({ }^{31} \mathrm{P}\right) \\
(\mathrm{ppm}) \\
\end{array}$ & $\begin{array}{c}\text { Trial 3 } \\
\Delta \delta\left({ }^{31} \mathrm{P}\right) \\
(\mathrm{ppm}) \\
\end{array}$ & $\begin{array}{c}\text { Trial } 4 \\
\Delta \delta\left({ }^{31} \mathrm{P}\right) \\
(\mathrm{ppm}) \\
\end{array}$ & $\begin{array}{c}\text { Avg } \\
\Delta \delta\left({ }^{31} \mathrm{P}\right) \\
(\mathrm{ppm}) \\
\end{array}$ & $\begin{array}{l}\text { Standard } \\
\text { Error of } \\
\text { the Mean }\end{array}$ \\
\hline \multicolumn{7}{|c|}{ Alkali and Alkaline Earth Metals } \\
\hline $\mathrm{LiBArF} \cdot \mathrm{OEt}_{2}$ & 8.7 & - & - & - & - & - \\
\hline $\mathrm{LiBF}_{4}$ & 7.5 & - & - & - & - & - \\
\hline $\mathrm{NaSbF}_{6}$ & 0.0 & - & - & - & - & - \\
\hline NaBArF & 6.4 & - & - & - & - & - \\
\hline $\mathrm{NaOTf}$ & 0.4 & 0.6 & - & - & 0.5 & - \\
\hline $\mathrm{NaBF}_{4}$ & 0.0 & 0.1 & - & - & 0.1 & - \\
\hline $\mathrm{MgCl}_{2}$ & 12.8 & - & - & - & - & - \\
\hline $\mathrm{MgBr}_{2}$ & 12.3 & - & - & - & - & - \\
\hline $\operatorname{Mg}(\mathrm{OTf})_{2}$ & 7.0 & 11.3 & - & - & 9.2 & - \\
\hline $\mathrm{Ca}(\mathrm{OTf})_{2}$ & 8.0 & - & - & - & - & - \\
\hline \multicolumn{7}{|l|}{ Rare Earth Metals } \\
\hline $\mathrm{Sc}(\mathrm{OTf})_{3}$ & 23.8 & 23.9 & 23.8 & 23.7 & 23.8 & 0.04 \\
\hline $\mathrm{ScCl}_{3}$ & 23.3 & 21.1 & - & - & 22.2 & - \\
\hline $\mathrm{ScCl}_{3}(\mathrm{THF})_{3}$ & 21.5 & - & - & - & - & - \\
\hline $\mathrm{Sc}(\mathrm{OTf})_{3}\left(\mathrm{H}_{2} \mathrm{O}\right)_{6}$ & 21.0 & - & - & - & - & - \\
\hline $\mathrm{Y}(\mathrm{OTf})_{3}$ & 17.1 & 17.1 & 17.5 & 17.4 & 17.3 & 0.09 \\
\hline $\mathrm{YCl}_{3}$ & 19.6 & - & - & - & - & - \\
\hline $\mathrm{La}(\mathrm{OTf})_{3}$ & 15.3 & 15.3 & 15.3 & - & 15.3 & 0.0 \\
\hline $\mathrm{LaCl}_{3}$ & 6.0 & - & - & - & - & - \\
\hline $\mathrm{CeCl}_{3}$ & 48.7 & 47.6 & 48.4 & - & 48.2 & 0.3 \\
\hline \multicolumn{7}{|l|}{ Transition Metals } \\
\hline $\mathrm{TiCl}_{4}$ & 44.6 & 45.3 & 45.1 & - & 45.0 & 0.2 \\
\hline $\operatorname{Ti}(\mathrm{OiPr})_{2}(\mathrm{OAr})_{2}{ }^{\mathrm{c}}$ & 25.3 & 25.2 & 25.3 & - & 25.3 & 0.03 \\
\hline $\mathrm{Hf}(\mathrm{OTf})_{4}$ & 39.0 & - & - & - & - & - \\
\hline $\mathrm{RuCl}_{3}$ & 1.6 & - & - & - & - & - \\
\hline AgOTf & 16.3 & - & - & - & - & - \\
\hline $\mathrm{AgSbF}_{6}$ & 20.3 & - & - & - & - & - \\
\hline $\mathrm{Zn}(\mathrm{OTf})_{2}$ & 21.3 & 20.2 & 21.3 & 21.8 & 21.2 & 0.3 \\
\hline $\mathrm{ZnCl}_{2}$ & 21.3 & 17.9 & 18.8 & - & 19.3 & 0.8 \\
\hline \multicolumn{7}{|l|}{ Post-Transition Metals } \\
\hline $\mathrm{Al}(\mathrm{OTf})_{3}$ & 20.7 & 18.7 & - & - & 19.7 & - \\
\hline $\mathrm{AlCl}_{3}$ & 27.8 & - & - & - & - & - \\
\hline $\operatorname{In}(\mathrm{OTf})_{3}$ & 27.2 & 25.5 & 24.5 & 24.3 & 25.4 & 0.6 \\
\hline $\mathrm{InCl}_{3}$ & 28.3 & 29.4 & 24.6 & - & 27.4 & 1.2 \\
\hline $\mathrm{Sn}(\mathrm{OTf})_{2}$ & 8.2 & - & - & - & - & - \\
\hline $\mathrm{SnCl}_{2}$ & 25.5 & - & - & - & - & - \\
\hline $\mathrm{SnCl}_{4}$ & 23.6 & 24.1 & - & - & 23.9 & - \\
\hline $\mathrm{Bi}(\mathrm{OTf})_{3}$ & 33.1 & 37.7 & 29.3 & 37.6 & 34.4 & 1.6 \\
\hline \multicolumn{7}{|l|}{ Boron/Metalloids } \\
\hline $\mathrm{BF}_{3} \cdot \mathrm{OEt}_{2}$ & 28.7 & - & - & - & - & - \\
\hline $\mathrm{B}\left(\mathrm{C}_{6} \mathrm{~F}_{5}\right)_{3}$ & 26.0 & - & - & - & - & - \\
\hline
\end{tabular}


${ }^{a}$ Binding experiments performed at $16 \mathrm{mM}$ final concentration of Lewis base with 3 equiv of Lewis acid with respect to phosphine oxide probe. The $\Delta \delta\left({ }^{31} \mathrm{P}\right)$ for TEPO-Lewis acid complexes were measured as the difference between the chemical shift of the complex in $\mathrm{CD}_{2} \mathrm{Cl}_{2}$ and the chemical shift of TEPO in $\mathrm{CD}_{2} \mathrm{Cl}_{2}(50.3 \mathrm{ppm})$. ${ }^{\mathrm{b}} \mathrm{Standard}$ Error of the Mean calculated using standard deviation/ $(\sqrt{ }($ sample size $))$. Standard errors were only calculated for Lewis acids with at least 3 measurements. ${ }^{\mathrm{c}}(\mathrm{OAr})_{2}=(S)$-BINOL

\section{IVb. ${ }^{31} \mathrm{P}$ NMR data for bisphosphine oxide probes TEMBP and MBPO}

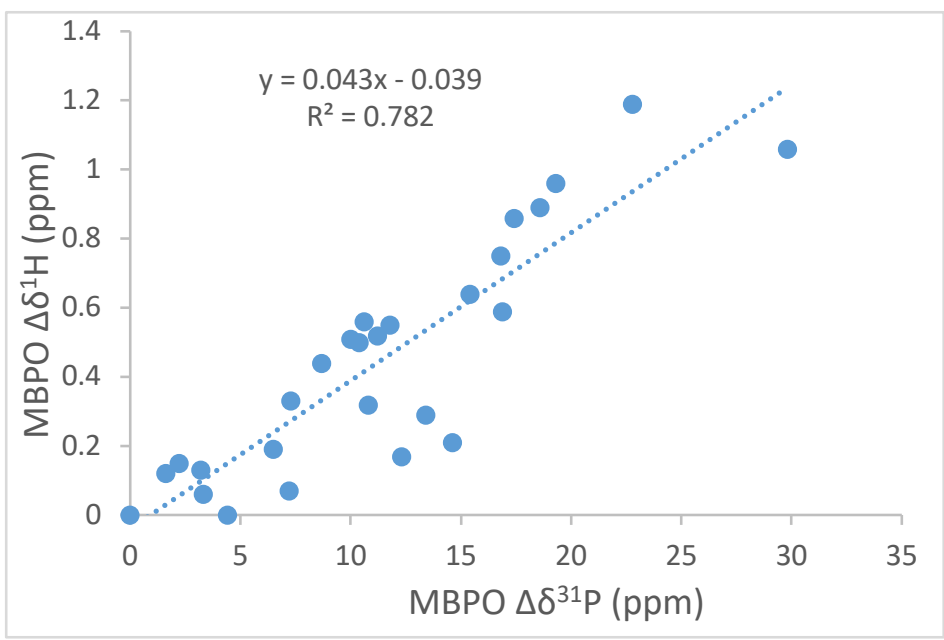

Figure S9. A linear correlation was observed between $\Delta \delta\left({ }^{1} \mathrm{H}\right)$ and $\Delta \delta\left({ }^{31} \mathrm{P}\right)$ NMR signals for MBPO. 
Table S9. $\Delta \delta\left({ }^{31} \mathrm{P}\right)$ NMR measurements for Lewis acid binding to ${ }^{31} \mathrm{P}$ NMR probes. ${ }^{\mathrm{a}}$

\begin{tabular}{|c|c|c|c|c|}
\hline Lewis acid & $\begin{array}{l}\text { TEPO } \\
\Delta \delta\left({ }^{31} \mathrm{P}\right) \\
(\mathrm{ppm})^{\mathrm{b}}\end{array}$ & $\begin{array}{c}\text { TEMBP } \\
\Delta \delta\left({ }^{31} \mathrm{P}\right) \\
(\mathrm{ppm})\end{array}$ & $\begin{array}{c}\text { MBPO } \\
\Delta \delta\left({ }^{31} \mathrm{P}\right) \\
(\mathrm{ppm})\end{array}$ & $\begin{array}{l}\text { MBPO } \\
\Delta \delta\left({ }^{1} \mathrm{H}\right) \\
(\mathrm{ppm})\end{array}$ \\
\hline \multicolumn{5}{|c|}{ Alkali and Alkaline Earth Metals } \\
\hline $\mathrm{LiBArF} \cdot \mathrm{OEt}_{2}$ & 8.7 & 1.9 & 8.9 & $-^{\mathrm{f}}$ \\
\hline $\mathrm{LiBF}_{4}$ & 7.5 & 1.6 & 3.3 & 0.06 \\
\hline NaOTf & 0.5 & 0.6 & 1.6 & 0.12 \\
\hline $\mathrm{NaBF}_{4}$ & 0.1 & 0.0 & 2.2 & 0.15 \\
\hline $\mathrm{NaBArF}$ & 6.4 & 1.0 & 6.5 & 0.19 \\
\hline $\mathrm{NaSbF}_{6}$ & 0.0 & 0.8 & 4.4 & 0.0 \\
\hline $\mathrm{MgBr}_{2}$ & 12.3 & 1.5 & 10.0 & 0.51 \\
\hline $\operatorname{Mg}(\mathrm{OTf})_{2}$ & 9.2 & 0.0 & $-\mathrm{e}$ & $-{ }^{f}$ \\
\hline $\mathrm{Ca}(\mathrm{OTf})_{2}$ & 8.0 & 2.4 & 7.3 & 0.33 \\
\hline \multicolumn{5}{|l|}{ Rare Earth Metals } \\
\hline $\mathrm{Sc}(\mathrm{OTf})_{3}$ & 23.8 & 2.7 & $17.4^{\mathrm{b}}$ & $0.86^{\mathrm{b}}$ \\
\hline $\mathrm{ScCl}_{3}$ & 22.2 & $2.9^{\mathrm{b}}$ & 19.2 & $-\mathrm{f}$ \\
\hline $\mathrm{ScCl}_{3}(\mathrm{THF})_{3}$ & 21.5 & $3.0^{\mathrm{b}}$ & 14.6 & 0.21 \\
\hline $\mathrm{Sc}(\mathrm{OTf})_{3}\left(\mathrm{H}_{2} \mathrm{O}\right)_{6}$ & 21.0 & 2.1 & 11.8 & 0.55 \\
\hline $\mathrm{Y}(\mathrm{OTf})_{3}$ & 17.3 & 1.9 & 10.6 & 0.56 \\
\hline $\mathrm{YCl}_{3}$ & 19.6 & 1.7 & 15.6 & $-\mathrm{f}$ \\
\hline $\mathrm{La}(\mathrm{OTf})_{3}$ & 15.3 & 2.2 & 8.7 & 0.44 \\
\hline $\mathrm{LaCl}_{3}$ & 6.0 & 1.5 & 8.7 & $-^{f}$ \\
\hline $\mathrm{CeCl}_{3}$ & 48.2 & $--^{e}$ & 22.7 & $-^{f}$ \\
\hline \multicolumn{5}{|l|}{ Transition Metals } \\
\hline $\mathrm{TiCl}_{4}$ & 45.0 & $4.1^{\mathrm{b}}$ & $29.8^{\mathrm{b}}$ & 1.06 \\
\hline $\mathrm{Ti}(\mathrm{OiPr})_{2}(\mathrm{OAr})_{2}{ }^{\mathrm{g}}$ & 25.3 & 3.8 & 12.3 & 0.17 \\
\hline $\mathrm{Hf}(\mathrm{OTf})_{4}$ & 39.0 & 2.2 & 10.4 & 0.50 \\
\hline $\mathrm{RuCl}_{3}$ & 1.6 & 0.0 & 0.0 & 0.00 \\
\hline $\mathrm{AgOTf}$ & 16.3 & 2.6 & 10.8 & 0.32 \\
\hline $\mathrm{AgSbF}_{6}$ & 20.3 & 2.8 & 13.4 & 0.29 \\
\hline $\mathrm{Zn}(\mathrm{OTf})_{2}$ & 21.2 & 2.8 & 11.2 & 0.52 \\
\hline $\mathrm{ZnCl}_{2}$ & 19.3 & 1.3 & 7.2 & 0.07 \\
\hline \multicolumn{5}{|c|}{ Post-Transition Metals } \\
\hline $\mathrm{Al}(\mathrm{OTf})_{3}$ & 19.7 & $-^{d}$ & 19.3 & 0.96 \\
\hline $\mathrm{AlCl}_{3}$ & 27.8 & 1.2 & $-\mathrm{e}$ & $-^{e}$ \\
\hline $\operatorname{In}(\mathrm{OTf})_{3}$ & 25.4 & 2.5 & 22.8 & 1.19 \\
\hline $\mathrm{InCl}_{3}$ & 27.4 & 2.2 & 15.6 & $--^{\mathrm{f}}$ \\
\hline $\mathrm{Sn}(\mathrm{OTf})_{2}$ & 8.2 & 2.9 & 18.6 & 0.89 \\
\hline $\mathrm{SnCl}_{2}$ & 25.5 & 1.3 & 16.8 & 0.75 \\
\hline $\mathrm{SnCl}_{4}$ & 23.9 & $-\mathrm{e}$ & 16.9 & 0.59 \\
\hline $\operatorname{Bi}(\mathrm{OTf})_{3}$ & 34.4 & 3.8 & 15.4 & 0.64 \\
\hline \multicolumn{5}{|l|}{ Boron/Metalloids } \\
\hline $\mathrm{BF}_{3} \cdot \mathrm{OEt}_{2}$ & 28.7 & 0.0 & $25.7,19.9$ & $1.03,0.91$ \\
\hline $\mathrm{B}\left(\mathrm{C}_{6} \mathrm{~F}_{5}\right)_{3}$ & 26.0 & 0.0 & 3.2 & 0.13 \\
\hline
\end{tabular}

${ }^{a}$ Data collected at $16.0 \mathrm{mM}$ as described in general procedure. ${ }^{b}$ Reported as average of triplicate experiments. ${ }^{\mathrm{c}}$ Average of two experiments. ${ }^{\mathrm{d}}$ Complex spectra with multiple peaks was observed. ${ }^{\mathrm{e}}$ No ${ }^{31} \mathrm{P}$ NMR signal was observed. ${ }^{\mathrm{f}} \mathrm{No}$ clear methylene peak was observed. ${ }^{\mathrm{g}}(\mathrm{OAr})_{2}=(S)-(-)-1,1^{\prime}-\mathrm{Bi}(2-$ naphthol). 


\section{V. ${ }^{31} \mathrm{P}$ NMR data for binding with metal-ligand complexes}

Pybox type ligands:

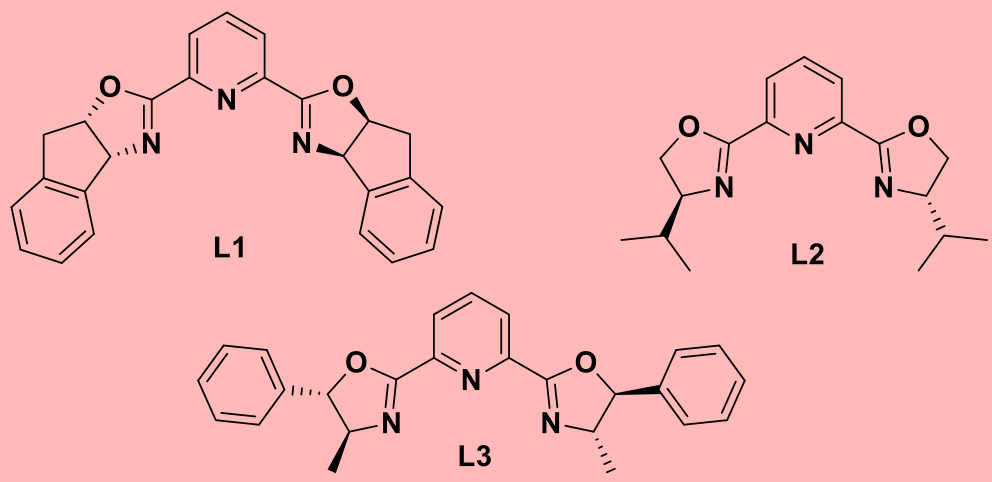

BINOL type ligands:

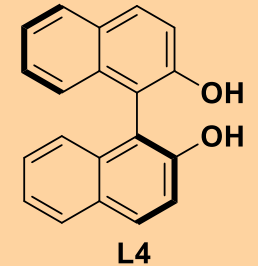

(S)-BINOL<smiles>Oc1c(-c2ccccc2)cc2ccccc2c1-c1c(-c2ccccc2)cc2ccccc2c1O</smiles>

L5

$(R)$-VANOL

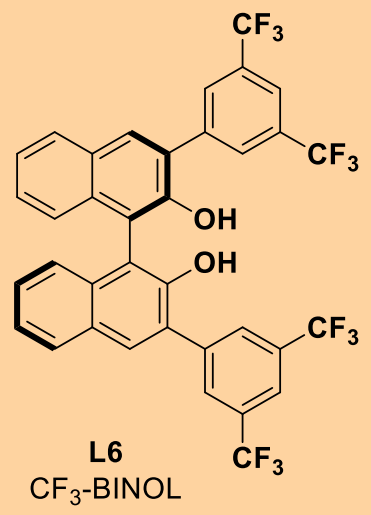

$\mathrm{CF}_{3}$-BINOL
Box type ligands:

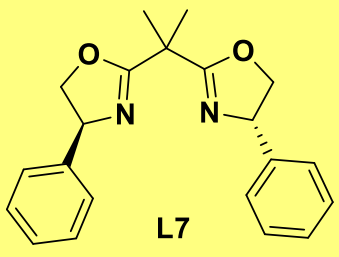

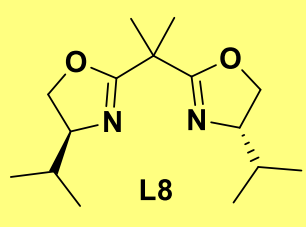

Pymox ligand:

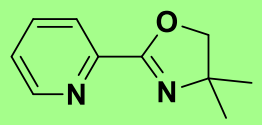

L9

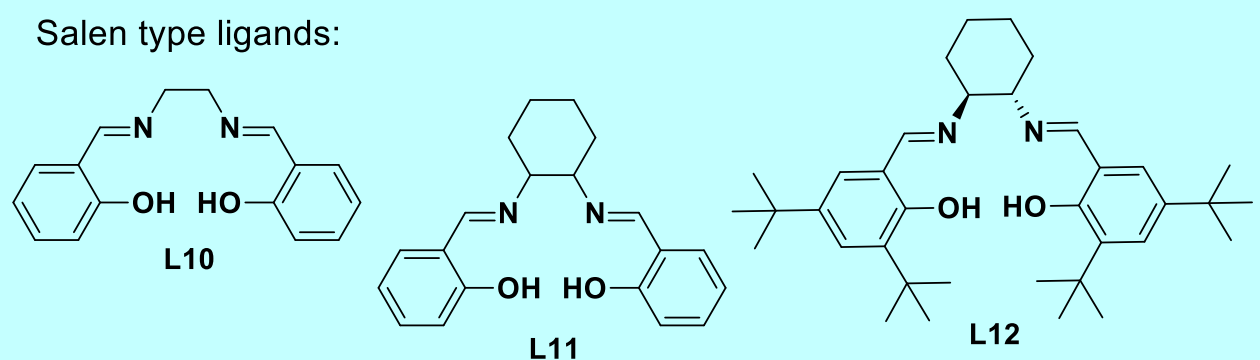

Figure S10. Ligands investigated in this work 
Table S10. $\Delta \delta\left({ }^{31} \mathrm{P}\right)$ NMR measurements for metal-ligand complexes binding to ${ }^{31} \mathrm{P}$ NMR probes. ${ }^{a}$

\begin{tabular}{|c|c|c|c|c|c|c|}
\hline Lewis acid & Ligand & Counterion & $\begin{array}{c}\text { TEPO } \\
\Delta \delta\left({ }^{31} \mathrm{P}\right) \\
(\mathrm{ppm})\end{array}$ & $\begin{array}{l}\text { TEMBP } \\
\Delta \delta\left({ }^{31} \mathrm{P}\right) \\
(\mathrm{ppm})\end{array}$ & $\begin{array}{l}\text { MBPO } \\
\Delta \delta\left({ }^{31} \mathrm{P}\right) \\
(\mathrm{ppm})\end{array}$ & $\begin{array}{l}\mathrm{MBPO} \\
\Delta \delta\left({ }^{1} \mathrm{H}\right) \\
(\mathrm{ppm})\end{array}$ \\
\hline $\mathrm{Sc}(\mathrm{OTf})_{3}$ & - & - & 23.8 & 2.5 & 17.4 & 0.86 \\
\hline $\mathrm{Sc}(\mathrm{OTf})_{3}$ & $(R, S)$-indapybox $(\mathbf{L 1})$ & - & 20.7 & 2.4 & 18.9 & 1.14 \\
\hline $\mathrm{Sc}(\mathrm{OTf})_{3}$ & L1 & $\mathrm{NaBArF}$ & 27.0 & 2.5 & 17.9 & 0.78 \\
\hline $\mathrm{Sc}(\mathrm{OTf})_{3}$ & L1 & $\mathrm{NaSbF}_{6}$ & 20.7 & 1.9 & 17.7 & 0.76 \\
\hline $\mathrm{Sc}(\mathrm{OTf})_{3}$ & L1 & $\mathrm{AgSbF}_{6}$ & 24.1 & 2.6 & 17.2 & 0.81 \\
\hline $\mathrm{Sc}(\mathrm{OTf})_{3}$ & - & $\mathrm{NaBArF}$ & 27.2 & 2.7 & 17.3 & 0.73 \\
\hline $\mathrm{Sc}(\mathrm{OTf})_{3}$ & - & $\mathrm{NaSbF}_{6}$ & 23.8 & 2.1 & 18.6 & 0.12 \\
\hline $\mathrm{Sc}(\mathrm{OTf})_{3}$ & - & $\mathrm{AgSbF}_{6}$ & 44.1 & 2.7 & 18.7 & 0.80 \\
\hline $\mathrm{Sc}(\mathrm{OTf})_{3}$ & $(4 S)$-isopropylpybox $(\mathbf{L 2})$ & - & 20.6 & 2.1 & 17.7 & 1.09 \\
\hline $\mathrm{Sc}(\mathrm{OTf})_{3}$ & $(S, S)$-norephedrinepybox $(\mathbf{L} \mathbf{3})$ & - & 20.6 & 2.2 & 17.6 & 0.86 \\
\hline $\mathrm{Sc}(\mathrm{OTf})_{3}$ & $(4 S)$-phenyl-box $(\mathbf{L} 7)$ & - & 17.6 & $-{ }^{d}$ & $-{ }^{d}$ & $-{ }^{d}$ \\
\hline $\mathrm{Sc}(\mathrm{OTf})_{3}$ & $(4 S)$-isopropyl-box $(\mathbf{L 8})$ & - & $-^{c}$ & $-d$ & 17.4 & $-d$ \\
\hline $\mathrm{Sc}(\mathrm{OTf})_{3}$ & dimethyl pymox (L9) & - & 20.3 & $-{ }^{d}$ & $18.5,17.2$ & $-{ }^{d}$ \\
\hline $\mathrm{Sc}(\mathrm{OTf})_{3}$ & Cy-Salen (L11) & - & 20.3 & $-{ }^{d}$ & $-\mathrm{d}$ & $-{ }^{d}$ \\
\hline $\mathrm{Sc}(\mathrm{OTf})_{3}$ & Jacobsen's Salen (L12) & - & $23.3,20.3$ & $-{ }^{d}$ & $-{ }^{d}$ & $-d^{d}$ \\
\hline $\mathrm{ScCl}_{3}(\mathrm{THF})_{3}$ & - & - & 21.5 & 3.0 & 14.6 & 0.21 \\
\hline $\mathrm{ScCl}_{3}(\mathrm{THF})_{3}$ & $\mathbf{L 1}$ & - & 19.5 & 1.1 & 13.4 & 0.19 \\
\hline $\mathrm{ScCl}_{3}(\mathrm{THF})_{3}$ & L1 & $\mathrm{NaBArF}$ & 22.1 & 2.8 & 14.5 & 0.18 \\
\hline $\operatorname{In}(\mathrm{OTf})_{3}$ & - & - & 25.3 & 2.7 & 22.8 & 1.19 \\
\hline $\operatorname{In}(\mathrm{OTf})_{3}$ & - & $\mathrm{NaSbF}_{6}$ & 25.4 & 2.5 & 22.4 & 1.17 \\
\hline $\operatorname{In}(\mathrm{OTf})_{3}$ & - & $\mathrm{NaBArF}$ & 39.7 & 2.0 & 23.1 & 1.18 \\
\hline $\operatorname{In}(\mathrm{OTf})_{3}$ & L1 & - & 22.8 & 0.9 & 22.5 & 1.16 \\
\hline $\mathrm{In}(\mathrm{OTf})_{3}$ & L1 & $\mathrm{NaSbF}_{6}$ & 24.4 & 0.4 & 22.6 & 0.92 \\
\hline $\operatorname{In}(\mathrm{OTf})_{3}$ & L1 & $\mathrm{AgSbF}_{6}$ & 22.7 & 0.7 & 22.7 & 0.86 \\
\hline $\operatorname{In}(\mathrm{OTf})_{3}$ & L1 & $\mathrm{NaBArF}$ & 21.4 & 2.7 & 22.7 & 1.05 \\
\hline $\mathrm{Zn}(\mathrm{OTf})_{2}$ & - & - & 21.8 & 2.8 & 13.2 & 0.52 \\
\hline $\mathrm{Zn}(\mathrm{OTf})_{2}$ & L1 & - & 21.5 & 2.2 & 11.1 & 0.51 \\
\hline $\mathrm{Zn}(\mathrm{OTf})_{2}$ & L1 & $\mathrm{NaBArF}$ & 22.2 & 2.7 & 11.2 & 0.51 \\
\hline $\mathrm{Zn}(\mathrm{OTf})_{2}$ & L1 & $\mathrm{NaSbF}_{6}$ & 21.7 & 2.7 & 11.0 & 0.51 \\
\hline $\mathrm{Zn}(\mathrm{OTf})_{2}$ & L1 & $\mathrm{AgSbF}_{6}$ & 22.4 & 2.7 & 11.0 & 0.29 \\
\hline $\mathrm{Zn}(\mathrm{OTf})_{2}$ & L7 & - & 19.4 & $-\mathrm{d}$ & $-d$ & $-\mathrm{d}$ \\
\hline $\mathrm{Zn}(\mathrm{OTf})_{2}$ & L8 & - & 20.7 & $-{ }^{d}$ & 13.6 & $-\mathrm{d}$ \\
\hline $\mathrm{Zn}(\mathrm{OTf})_{2}$ & L9 & - & 2.4 & $-d$ & 11.2 & $\mathrm{~d}^{\mathrm{d}}$ \\
\hline $\mathrm{Zn}(\mathrm{OTf})_{2}$ & L11 & - & 21.7 & $-{ }^{d}$ & $-\mathrm{d}$ & $-\mathrm{d}$ \\
\hline $\mathrm{Zn}(\mathrm{OTf})_{2}$ & L12 & - & 21.5 & $-{ }^{d}$ & $-{ }^{d}$ & $-\mathrm{d}$ \\
\hline $\mathrm{Ca}(\mathrm{OTf})_{2}$ & - & - & 8.0 & 2.4 & 7.3 & 0.33 \\
\hline $\mathrm{Ca}(\mathrm{OTf})_{2}$ & L1 & - & 7.7 & 1.0 & 7.3 & 0.13 \\
\hline $\mathrm{Ca}(\mathrm{OTf})_{2}$ & L1 & $\mathrm{NaBArF}$ & 9.3 & 2.4 & 8.2 & 0.48 \\
\hline- & - & $\mathrm{NaSbF}_{6}$ & 0.0 & 0.8 & 4.4 & 0.0 \\
\hline- & - & $\mathrm{AgSbF}_{6}$ & 20.3 & 2.8 & 13.4 & 0.29 \\
\hline- & - & $\mathrm{NaBArF}$ & 6.4 & 1.0 & 6.5 & 0.19 \\
\hline $\mathrm{Ti}(\mathrm{O} i-\mathrm{Pr})_{4}$ & - & - & 0.0 & 0.0 & 0.0 & 0.00 \\
\hline $\mathrm{Ti}(\mathrm{O} i-\mathrm{Pr})_{4}$ & $(S)$-BINOL $(\mathbf{L} 4)^{\mathrm{b}}$ & - & 25.3 & 3.8 & 12.3 & 0.17 \\
\hline $\mathrm{Ti}(\mathrm{O} i-\mathrm{Pr})_{4}$ & $(R)$-VANOL $(\mathbf{L 5})$ & - & 20.4 & 3.9 & 10.9 & 0.15 \\
\hline $\mathrm{Ti}(\mathrm{O} i-\mathrm{Pr})_{4}$ & $\mathrm{CF}_{3}$-BINOL (L6) & - & 22.2 & 0.1 & 17.8 & $-{ }^{e}$ \\
\hline
\end{tabular}


${ }^{\mathrm{a}}$ Data collected according to General procedure for binding with metal-ligand complexes. ${ }^{\mathrm{b}}$ In the absence of $\mathrm{Ti}(\mathrm{O} i-\mathrm{Pr})_{4}$ the $\Delta \delta\left({ }^{31} \mathrm{P}\right)$ for binding of $(S)$-BINOL with TEPO is $3.8 \mathrm{ppm}$. Binding experiments with $\mathrm{Ti}(\mathrm{O} i \text {-Pr })_{4} /(\mathrm{R})-\mathrm{BINOL}$ complexes also resulted in $\Delta \delta\left({ }^{31} \mathrm{P}\right)=25.3$, however racemic mixtures resulted in decreased $\Delta \delta\left({ }^{31} \mathrm{P}\right)(20.6 \mathrm{ppm}) .{ }^{\mathrm{c}} \mathrm{No}{ }^{31} \mathrm{P}$ signal observed. ${ }^{\mathrm{d}} \Delta \delta\left({ }^{31} \mathrm{P}\right)$ not measured with this probe. ${ }^{\mathrm{e}}$ No clear methylene peak was observed.

\section{Ligand binding data}

In addition to $(R, S)$-indapybox (L1) and binaphthol complexes, preliminary binding data was acquired for pyMOX, BOX and Salen type ligands. In general, these ligand complexes have decreased $\Delta \delta\left({ }^{31} \mathrm{P}\right)$ values upon probe binding compared to Lewis acids alone.

$\Delta \delta\left({ }^{31} \mathrm{P}\right)$ upon TEPO binding and signal intensity decreases upon the addition of $\mathbf{L} \mathbf{1}$ to $\mathrm{Sc}(\mathrm{OTf})_{3}$ (Figure 11). A decrease in signal intensity and $\Delta \delta\left({ }^{31} \mathrm{P}\right)$ are observed with the addition of 1.1 equivalents of $\mathbf{L 1}$ to $\mathrm{Sc}(\mathrm{OTf})_{3}$. Upon addition of $>1.1$ equiv of $\mathbf{L 1}$, significant signal broadening and a decrease in signal intensity indicate that multiple species are present, indicating multiple binding modes competing for TEPO binding to the scandium metal. $\Delta \delta\left({ }^{31} \mathrm{P}\right)$ upon TEPO binding and signal intensity decreases upon the addition of $\mathbf{L 1}$ to $\mathrm{Zn}(\mathrm{OTf})_{2}$ (Figure 12). A decrease in signal intensity and $\Delta \delta\left({ }^{31} \mathrm{P}\right)$ are observed with the addition of 1.1 equivalents of $\mathbf{L 1}$ to $\mathrm{Zn}(\mathrm{OTf})_{2}$. Upon addition of greater than 1.1 equiv of $\mathbf{L 1}$, a sharp peak is observed at $\Delta \delta\left({ }^{31} \mathrm{P}\right)=0.0 \mathrm{ppm}$, indicating that TEPO is no longer binding to the metal-ligand complex. 


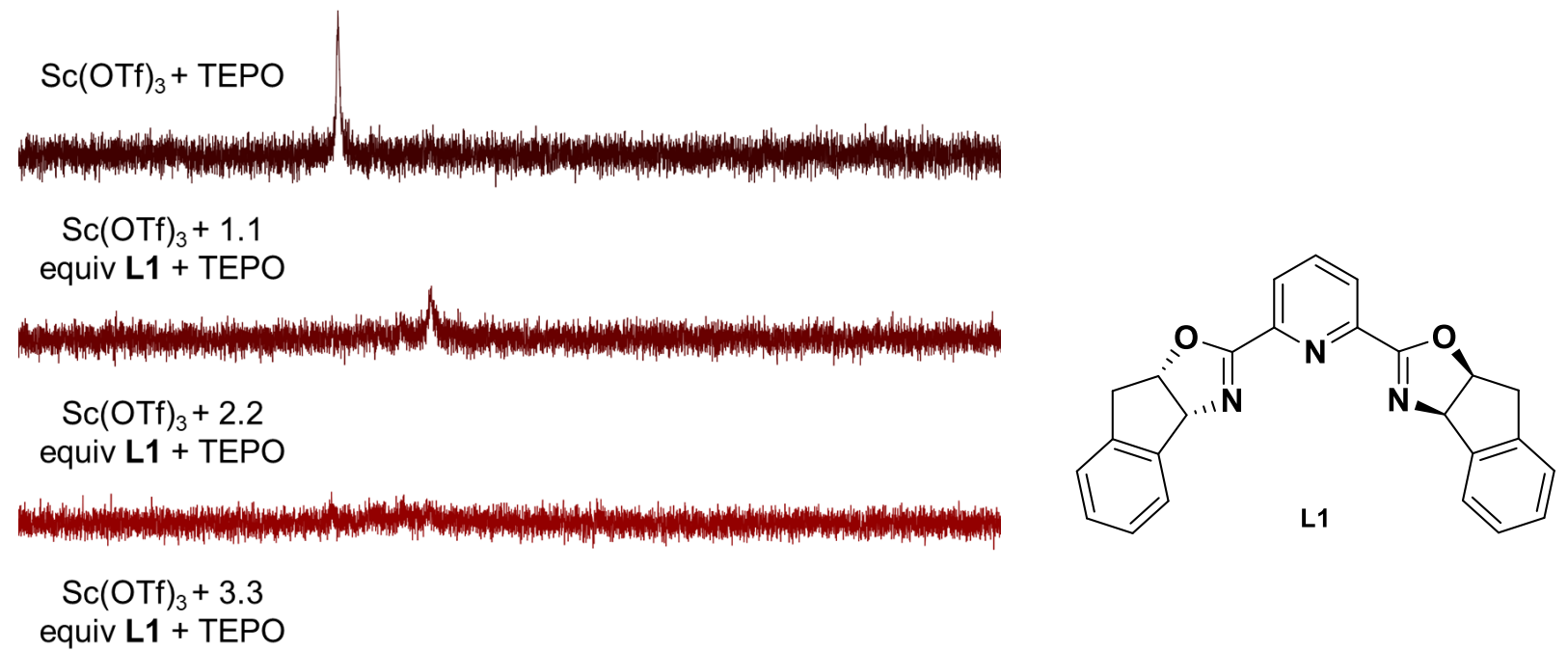

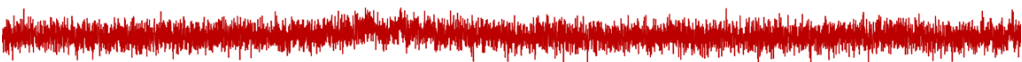

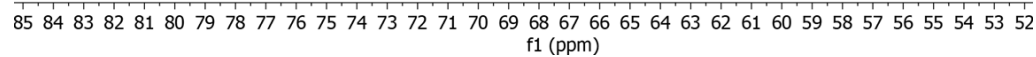

Figure S11. Effect of ligand stoichiometry on ${ }^{31} \mathrm{P}$ data for $\mathrm{TEPO} / \mathrm{Sc}(\mathrm{OTf})_{3} / \mathbf{L 1}$ mixtures.

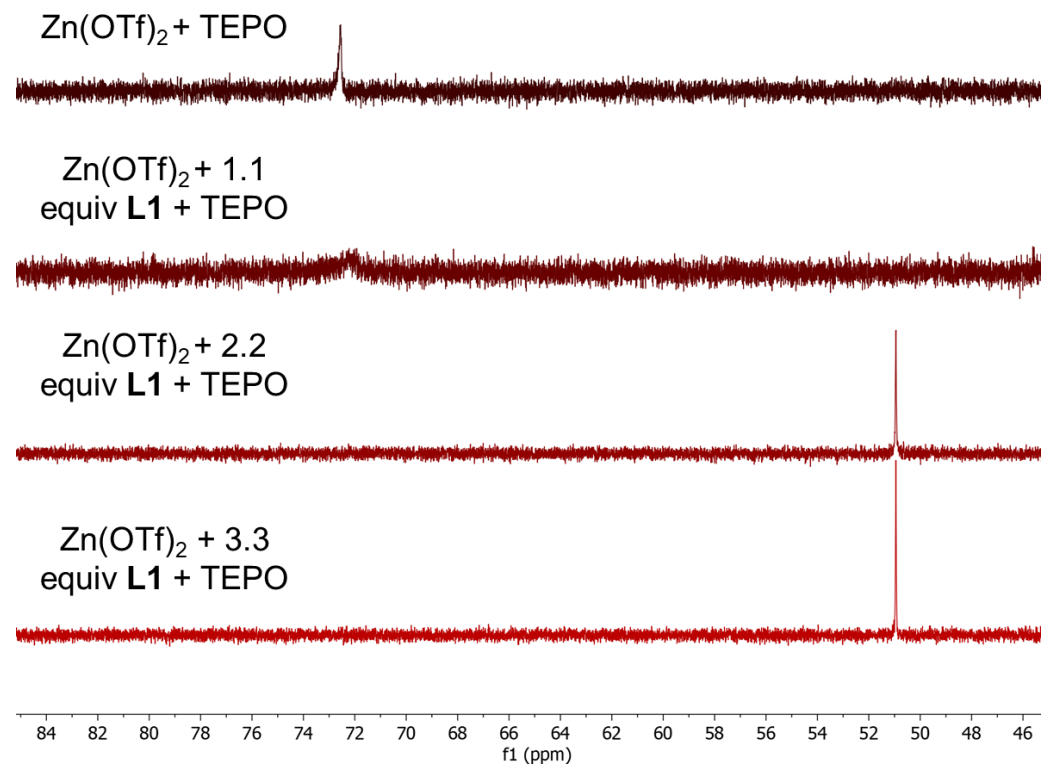

Figure S12. Effect of ligand stoichiometry on ${ }^{31} \mathrm{P}$ data for $\mathrm{TEPO} / \mathrm{Zn}(\mathrm{OTf})_{2} / \mathbf{L 1}$ mixtures. 


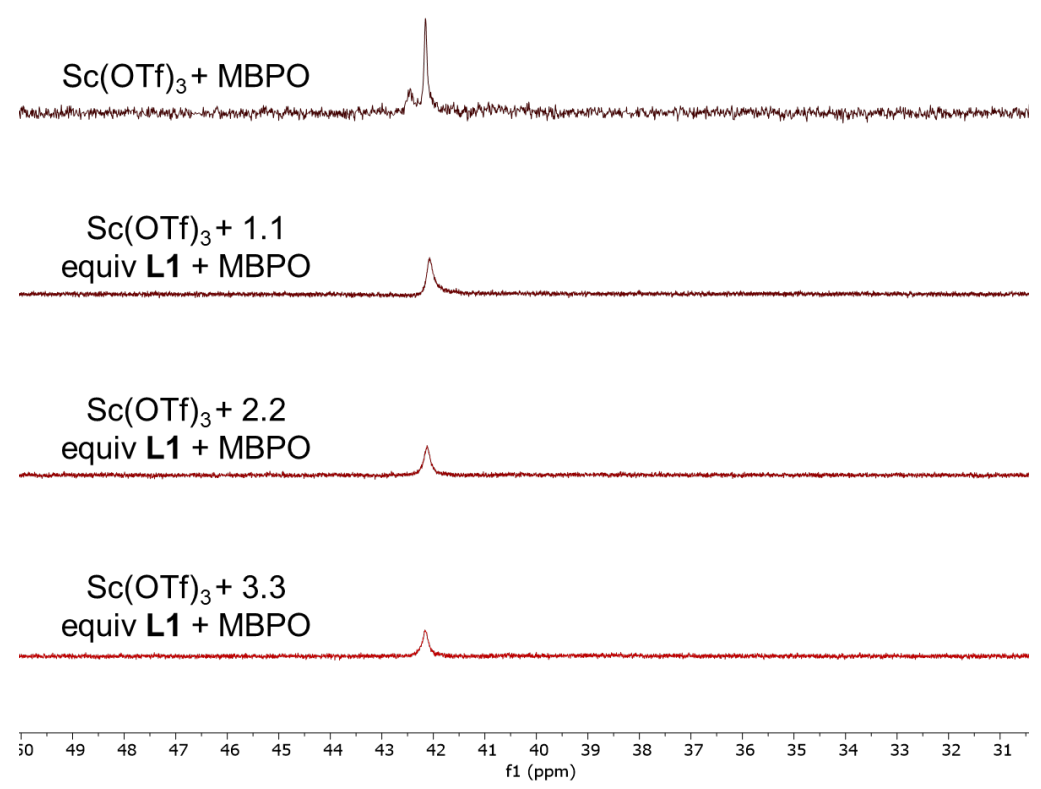

Figure S13. Effect of ligand stoichiometry on ${ }^{31} \mathrm{P}$ data for $\mathrm{MBPO} / \mathrm{Sc}(\mathrm{OTf})_{3} / \mathbf{L} 1$ mixtures.

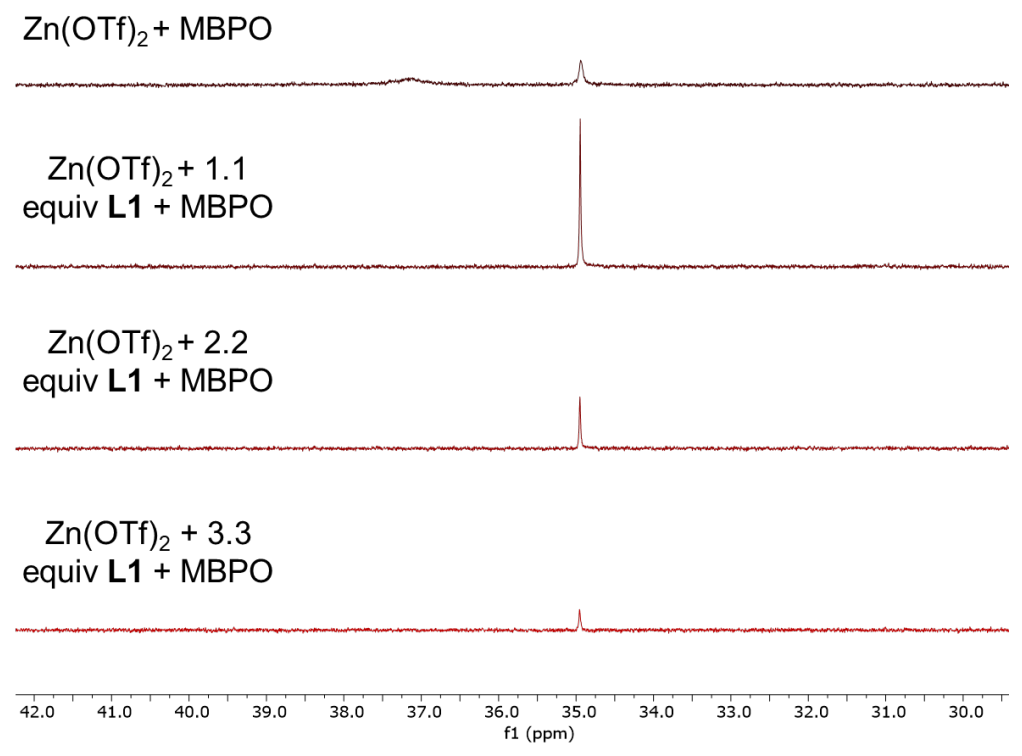

Figure S14. Effect of ligand stoichiometry on ${ }^{31} \mathrm{P}$ data for $\mathrm{MBPO} / \mathrm{Zn}(\mathrm{OTf})_{2} / \mathbf{L 1}$ mixtures.

$\Delta \delta\left({ }^{31} \mathrm{P}\right)$ signal intensity decreases for TEPO binding upon the addition of $\mathbf{L} 7$ to $\mathrm{Sc}(\mathrm{OTf})_{3}$. At 1.1 equiv of ligand, a decreased $\Delta \delta\left({ }^{31} \mathrm{P}\right)$ value is observed. When additional ligand is added, the signal disappears/becomes too broad to observe. Only a slight bump in the baseline can be observed. $\Delta \delta\left({ }^{31} \mathrm{P}\right)$ upon TEPO binding and signal intensity decreases upon the addition of $\mathbf{L} 7$ to $\mathrm{Zn}(\mathrm{OTf})_{2}$. A similar effect is observed with $\mathrm{Zn}(\mathrm{OTf})_{2}$, although the ${ }^{31} \mathrm{P}$ NMR signal is decreased and further broadened at 1.1 equivalent of ligand. 


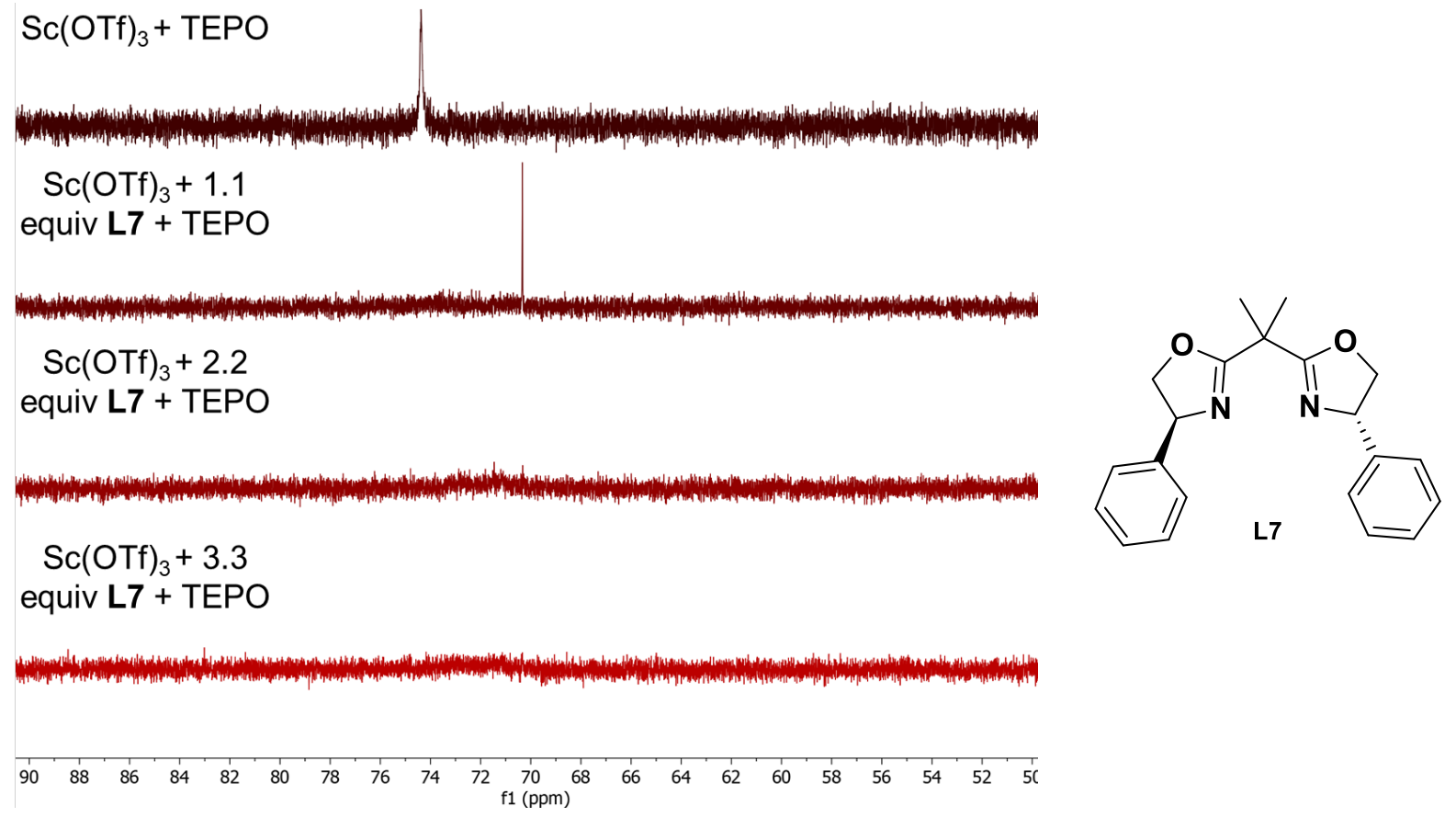

Figure S15. Effect of ligand stoichiometry on ${ }^{31} \mathrm{P}$ data for $\mathrm{TEPO} / \mathrm{Sc}(\mathrm{OTf})_{3} / \mathbf{L} 7$ mixtures.

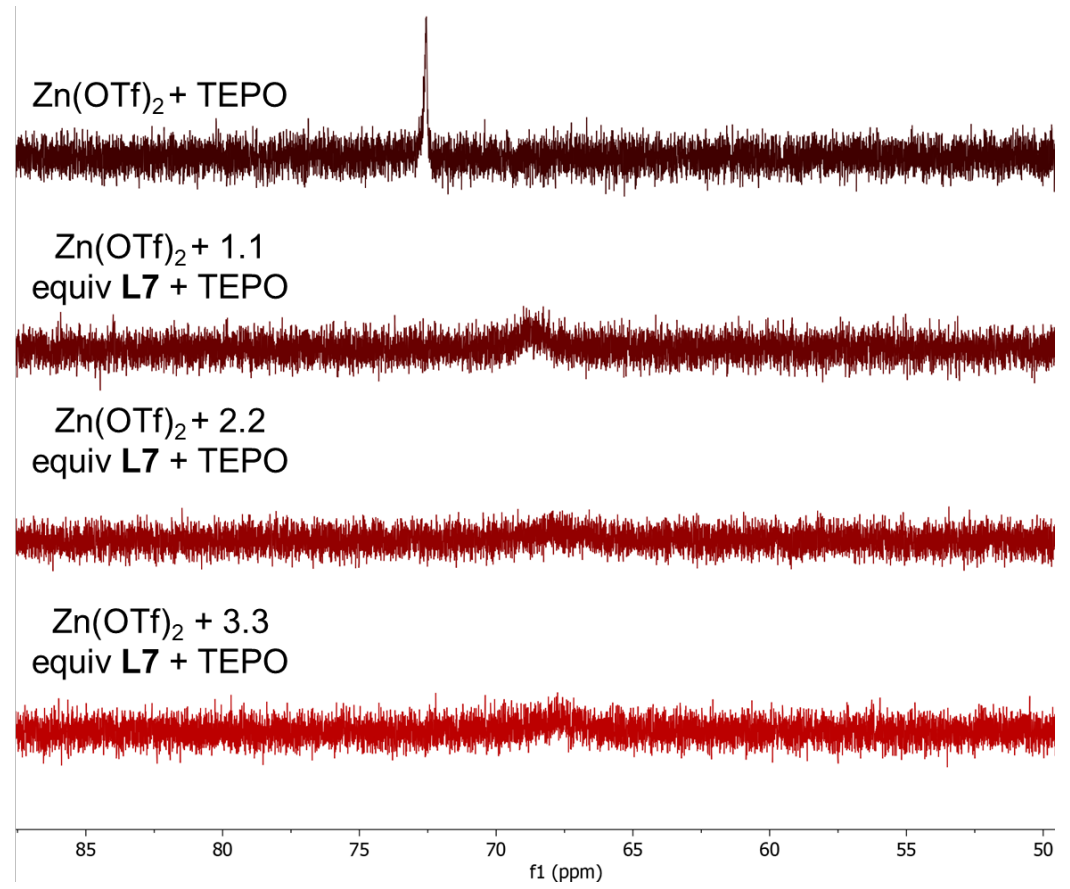

Figure S16. Effect of ligand stoichiometry on ${ }^{31} \mathrm{P}$ data for TEPO/Zn $(\mathrm{OTf})_{2} / \mathbf{L} 7$ mixtures.

$\Delta \delta\left({ }^{31} \mathrm{P}\right)$ upon TEPO binding and signal intensity decreases upon the addition of $\mathbf{L 8}$ to $\mathrm{Sc}(\mathrm{OTf})_{3}$. With $\mathbf{L 8}$, a lack of signal is observed with 1.1 equivalent of ligand. An increased signal is observed with 2.2 equiv, although more than one species appears to be present in solution. The signal again decreases with 3.3 equiv of L8. $\Delta \delta\left({ }^{31} \mathrm{P}\right)$ signal intensity upon TEPO binding decreases upon the addition of $\mathbf{L 8}$ to $\mathrm{Zn}(\mathrm{OTf})_{2}$. 
With $\mathrm{Zn}(\mathrm{OTf})_{2}$, a decrease in signal intensity and $\Delta \delta\left({ }^{31} \mathrm{P}\right)$ value is observed upon addition of 1.1 equivalents of ligand. Increasing the equivalents of $\mathbf{L 8}$ to 2.2 increases the signal intensity, although the $\Delta \delta\left({ }^{31} \mathrm{P}\right)$ does not change. When 3.3 equivalents of $\mathbf{L 8}$ are used, the $\Delta \delta\left({ }^{31} \mathrm{P}\right)$ value decreases and the signal is broadened.

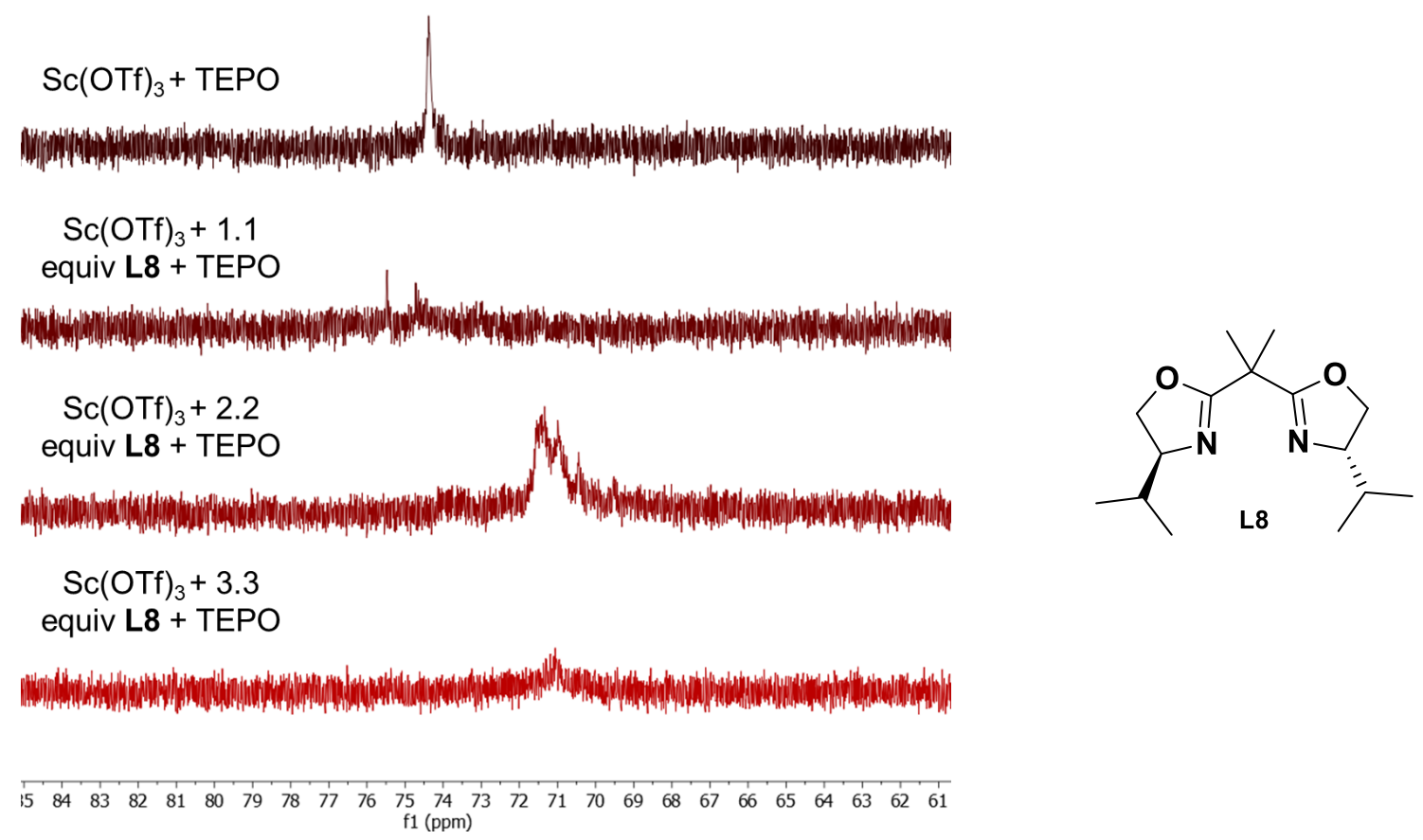

Figure S17. Effect of ligand stoichiometry on ${ }^{31} \mathrm{P}$ data for $\mathrm{TEPO} / \mathrm{Sc}(\mathrm{OTf})_{3} / \mathbf{L 8}$ mixtures.

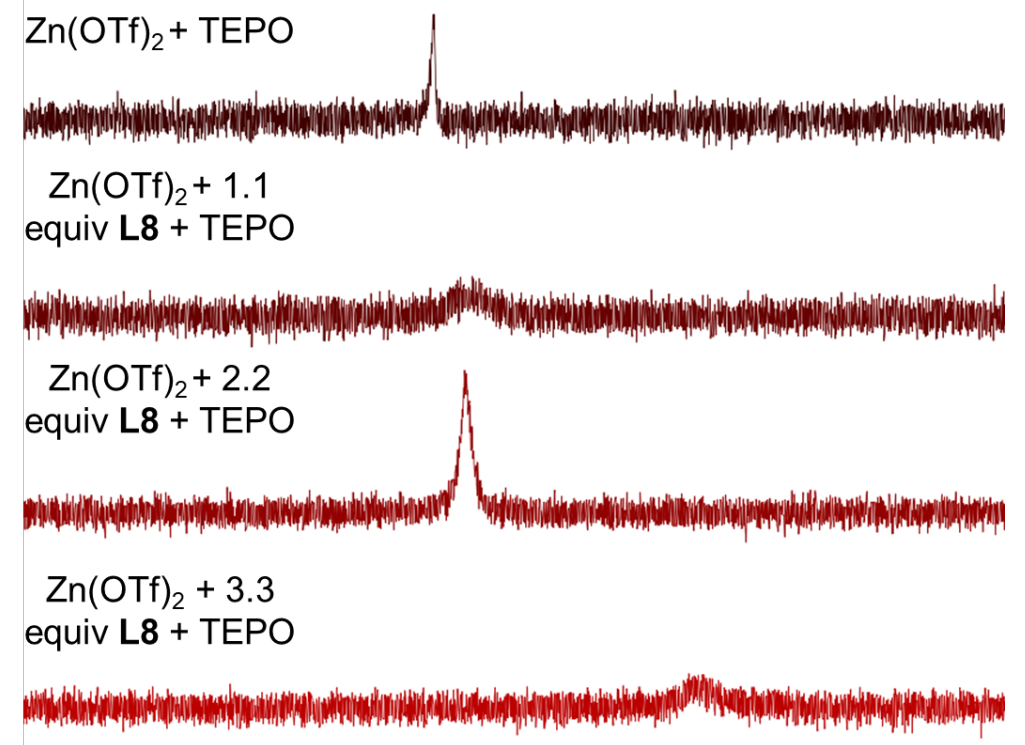

\begin{tabular}{lllllllllllllllllllllllllllll}
\hline 82 & 81 & 80 & 79 & 78 & 77 & 76 & 75 & 74 & 73 & 72 & 71 & 70 & 69 & 68 & 67 & 66 & 65 & 64 & 63 & 62 & 61 & 60 & 59
\end{tabular}

Figure S18. Effect of ligand stoichiometry on ${ }^{31} \mathrm{P}$ data for $\mathrm{TEPO} / \mathrm{Zn}(\mathrm{OTf})_{2} / \mathbf{L 8}$ mixtures. 


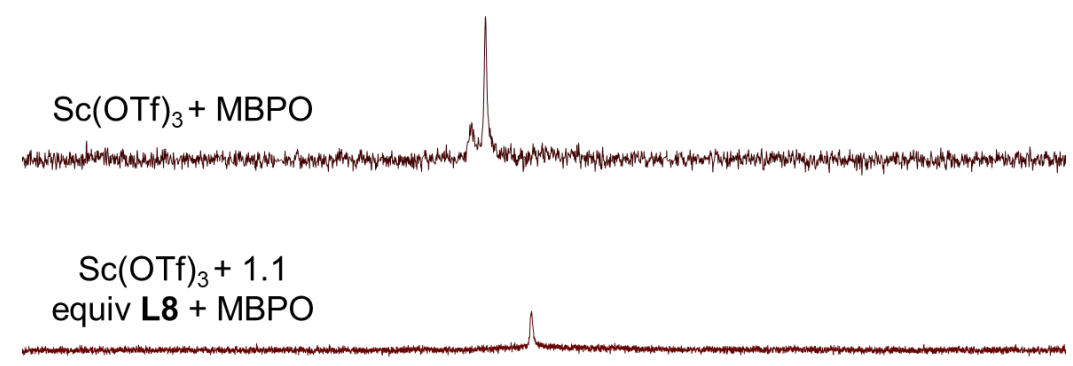

$\mathrm{Sc}(\mathrm{OTf})_{3}+2.2$

equiv L8 + MBPO

$\mathrm{Sc}(\mathrm{OTf})_{3}+3.3$

equiv L8 + MBPO

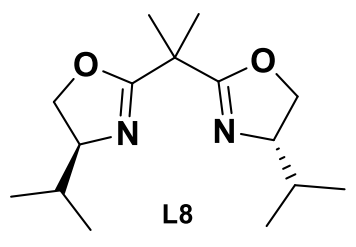

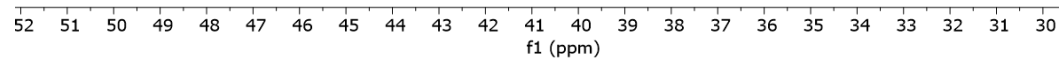

Figure S19. Effect of ligand stoichiometry on ${ }^{31} \mathrm{P}$ data for $\mathrm{MBPO} / \mathrm{Sc}(\mathrm{OTf})_{3} / \mathbf{L 8}$ mixtures.

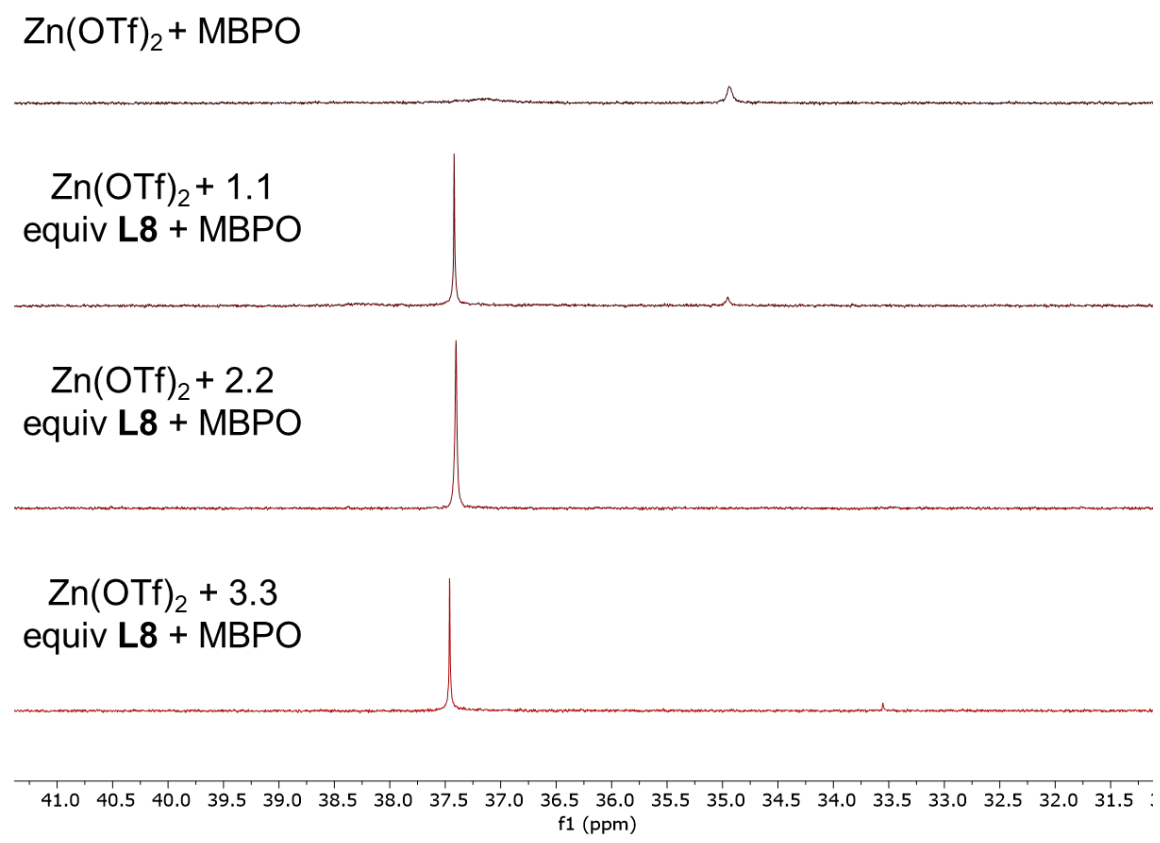

Figure S20. Effect of ligand stoichiometry on ${ }^{31} \mathrm{P}$ data for $\mathrm{MBPO} / \mathrm{Zn}(\mathrm{OTf})_{3} / \mathbf{L 8}$ mixtures 


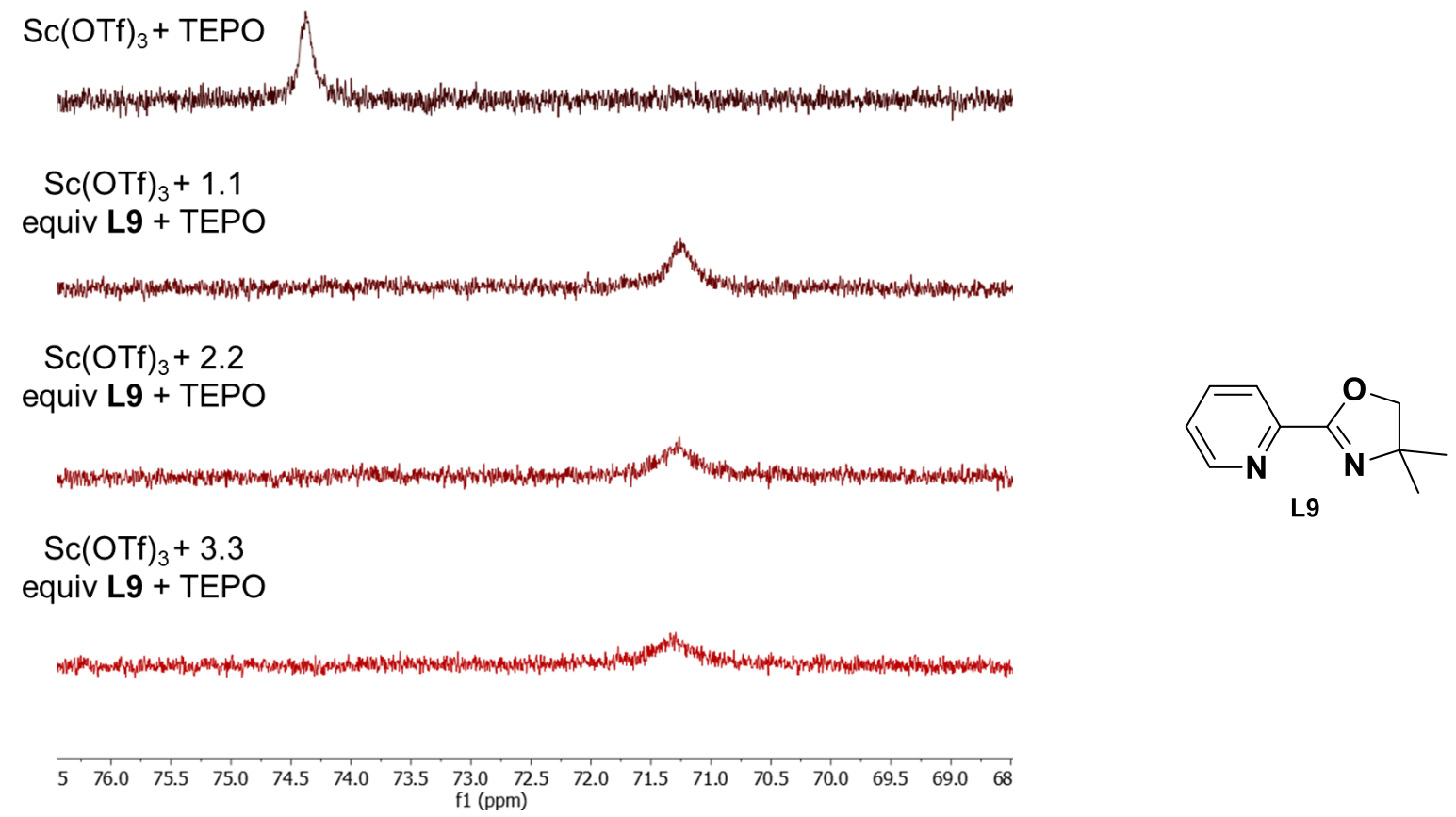

Figure S21. Effect of ligand stoichiometry on ${ }^{31} \mathrm{P}$ data for $\mathrm{TEPO} / \mathrm{Sc}(\mathrm{OTf})_{3} / \mathbf{L 9}$ mixtures

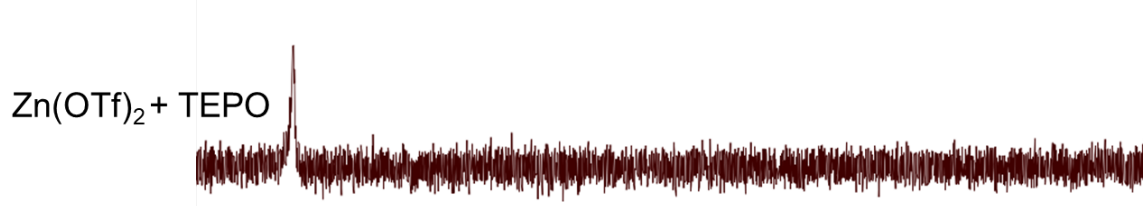

$\mathrm{Zn}(\mathrm{OTf})_{2}+1.1$

equiv L9 + TEPO

$\mathrm{Zn}(\mathrm{OTf})_{2}+2.2$

equiv L9 + TEPO

$\mathrm{Zn}(\mathrm{OTf})_{2}+3.3$

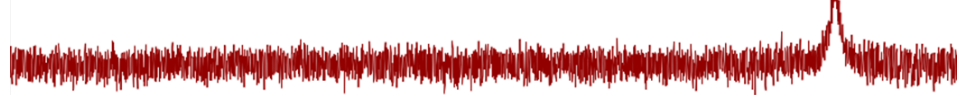

equiv L9 + TEPO

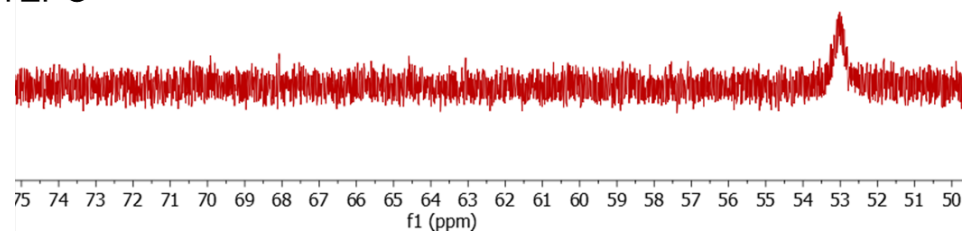

Figure S22. Effect of ligand stoichiometry on ${ }^{31} \mathrm{P}$ data for TEPO/Zn $(\mathrm{OTf})_{2} / \mathbf{L 9}$ mixtures 

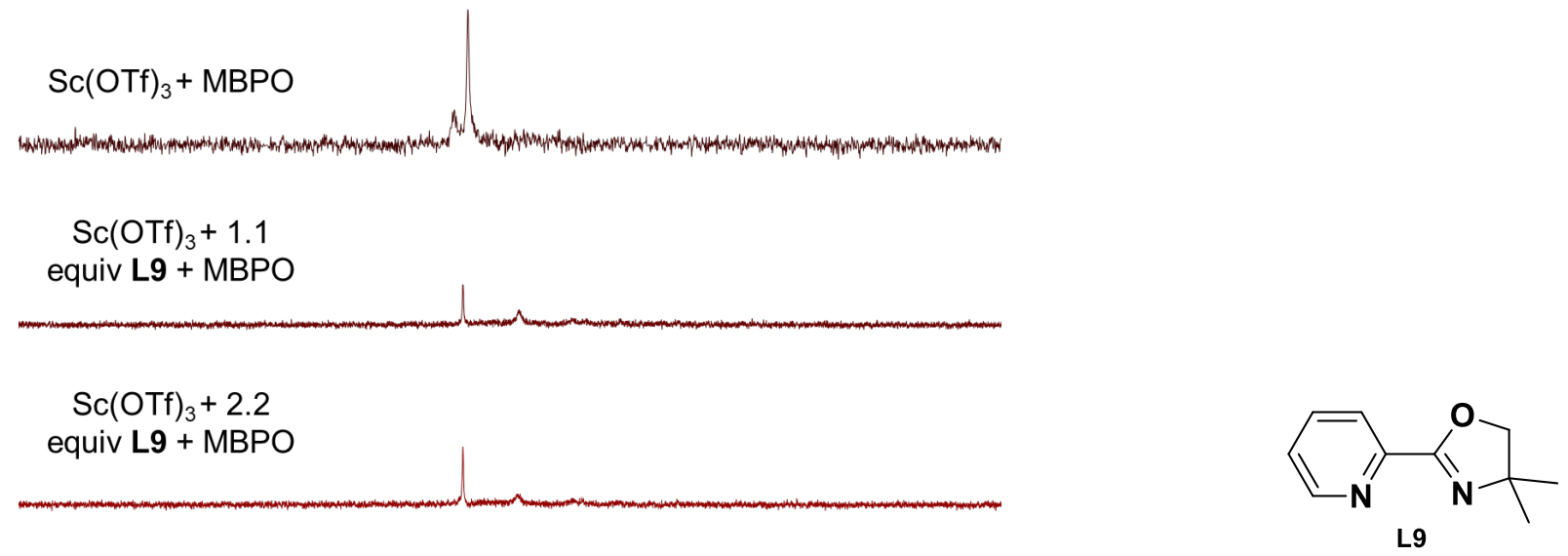

$$
\mathrm{Sc}(\mathrm{OTf})_{3}+3.3
$$

equiv L9 + MBPO

$\begin{array}{llllllllllllllllllllll}51 & 50 & 49 & 48 & 47 & 46 & 45 & 44 & 43 & 42 & \begin{array}{c}41 \\ \mathrm{f} 1(\mathrm{ppm})\end{array} & 39 & 38 & 37 & 36 & 35 & 34 & 33 & 32 & 31\end{array}$

Figure S23. Effect of ligand stoichiometry on ${ }^{31} \mathrm{P}$ data for $\mathrm{MBPO} / \mathrm{Sc}(\mathrm{OTf})_{3} / \mathbf{L} \mathbf{9}$ mixtures.

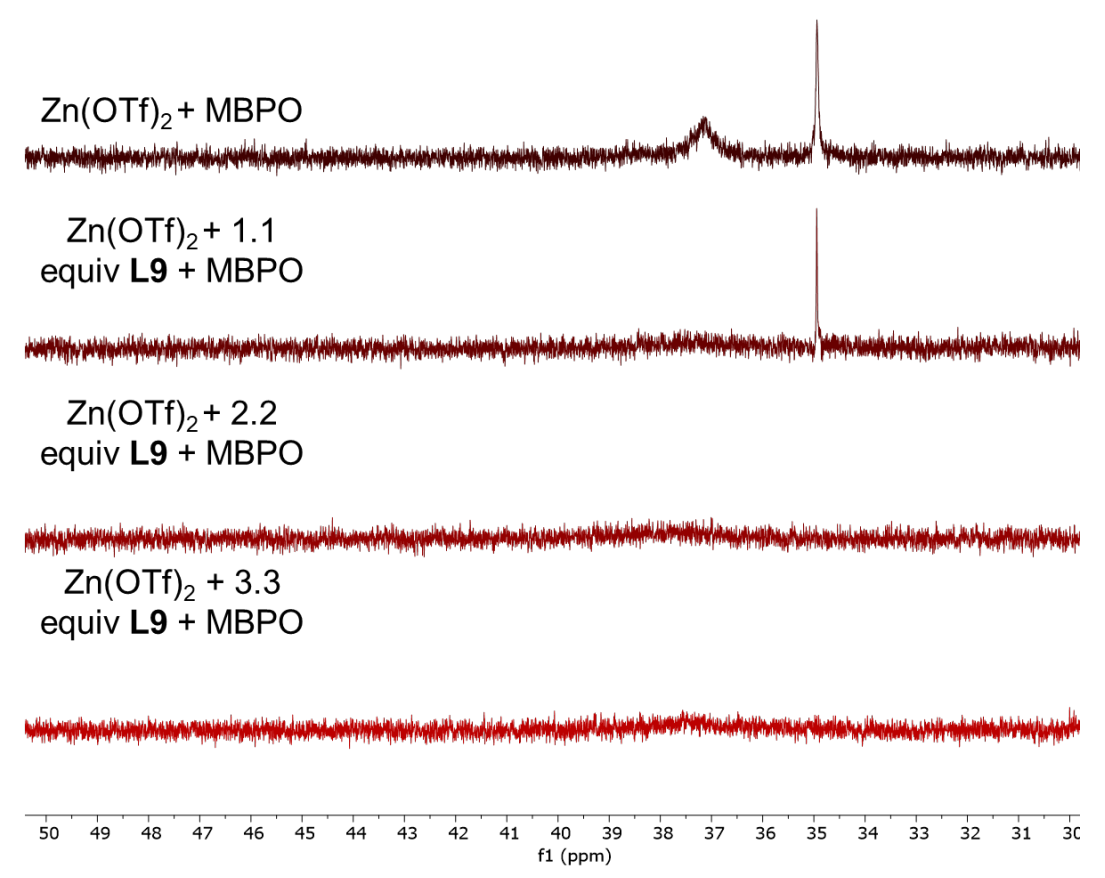

Figure S24. Effect of ligand stoichiometry on ${ }^{31} \mathrm{P}$ data for $\mathrm{MBPO} / \mathrm{Zn}(\mathrm{OTf})_{2} / \mathbf{L} 9$ mixtures 


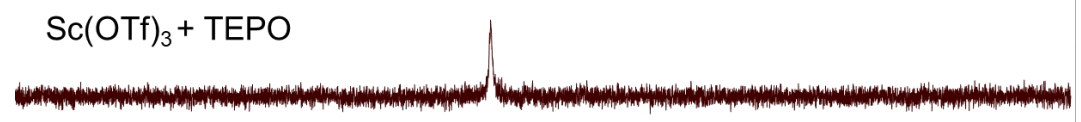

$\mathrm{Sc}(\mathrm{OTf})_{3}+1.1$

equiv L11 + TEPO

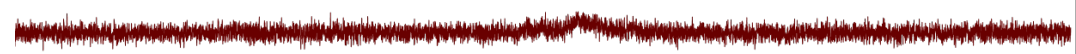

$\mathrm{Sc}(\mathrm{OTf})_{3}+2.2$ equiv L11 + TEPO

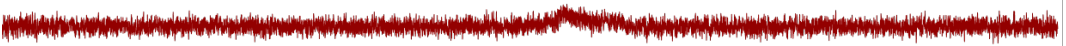

$\mathrm{Sc}(\mathrm{OTf})_{3}+3.3$

equiv L11 + TEPO

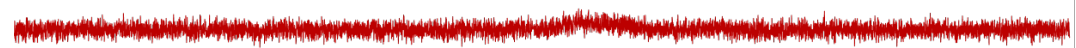

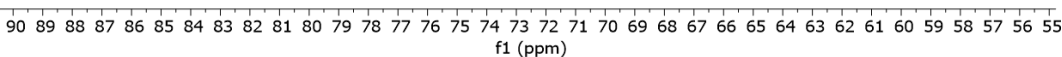

Figure S25. Effect of ligand stoichiometry on ${ }^{31} \mathrm{P}$ data for TEPO/Sc(OTf $)_{3} / \mathbf{L 1 1}$ mixtures

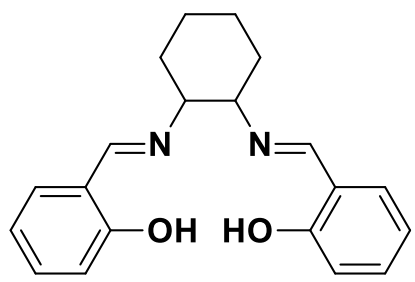

L11

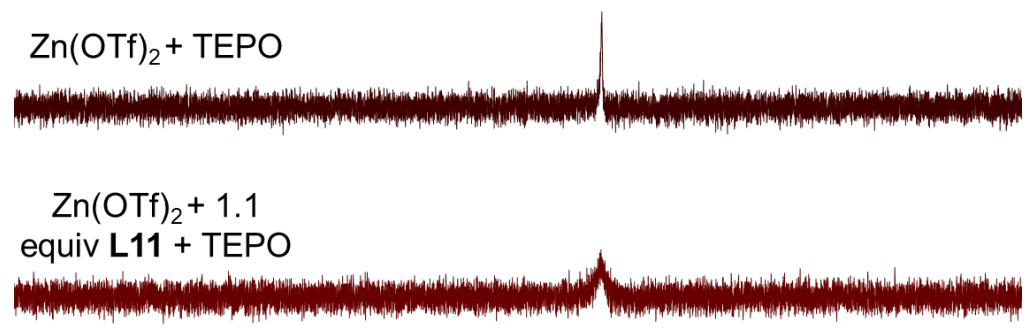

$\mathrm{Zn}(\mathrm{OTf})_{2}+2.2$

equiv L11 + TEPO
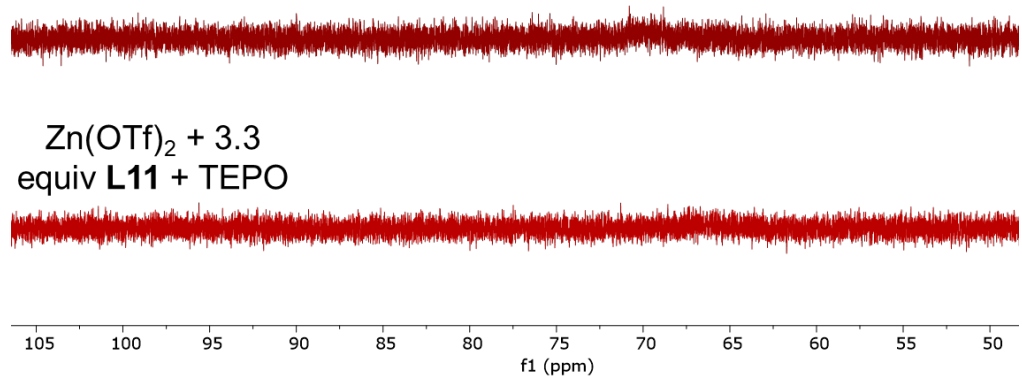

Figure S26. Effect of ligand stoichiometry on ${ }^{31} \mathrm{P}$ data for $\mathrm{TEPO} / \mathrm{Zn}(\mathrm{OTf})_{2} / \mathbf{L 1 1}$ mixtures 
$\Delta \delta\left({ }^{31} \mathrm{P}\right)$ signal intensity upon TEPO binding decreases upon the addition of $\mathbf{L 1 2}$ to $\operatorname{Sc}(\mathrm{OTf})_{3}$. With the very sterically demanding Jacobsen Salen ligand, a titration effect is observed. Upon addition of 1.1 equiv of L12, two peaks are observed: one peak that corresponds to unbound $\operatorname{Sc}(\mathrm{OTf})_{3}$ and another peak at a decreased $\Delta \delta\left({ }^{31} \mathrm{P}\right)$ shift. Upon addition of 2.2 equiv of L12, a third peak with an even smaller $\Delta \delta\left({ }^{31} \mathrm{P}\right)$ value is observed in addition to the two peaks observed at 1.1 equiv of L12. At 3.3 equiv of L12, the peak corresponding to unbound $\mathrm{Sc}(\mathrm{OTf})_{3}$ has disappeared and only the two peaks with lower $\Delta \delta\left({ }^{31} \mathrm{P}\right)$ values are observed. $\Delta \delta\left({ }^{31} \mathrm{P}\right)$ signal intensity upon TEPO binding decreases upon the addition of $\mathbf{L 1 2}$ to $\mathrm{Zn}(\mathrm{OTf})_{2}$. A titration effect is also observed with $\mathrm{Zn}(\mathrm{OTf})_{2}$. With the addition of 1.1 equiv L12, most of the $\mathrm{Zn}(\mathrm{OTf})_{2}$ appears to be unbound with a slight additional peak observed at a slightly lower $\Delta \delta\left({ }^{31} \mathrm{P}\right)$ than $\mathrm{Zn}(\mathrm{OTf})_{2}$ alone. This second lower ppm peak increases in intensity and the unbound $\mathrm{Zn}(\mathrm{OTf})_{2}$ peak decreases in intensity as the equivalents of $\mathbf{L 1 2}$ are increased. At 3.3 equiv of L12, only a slight peak indicating unbound $\mathrm{Zn}(\mathrm{OTf})_{2}$ is observed.

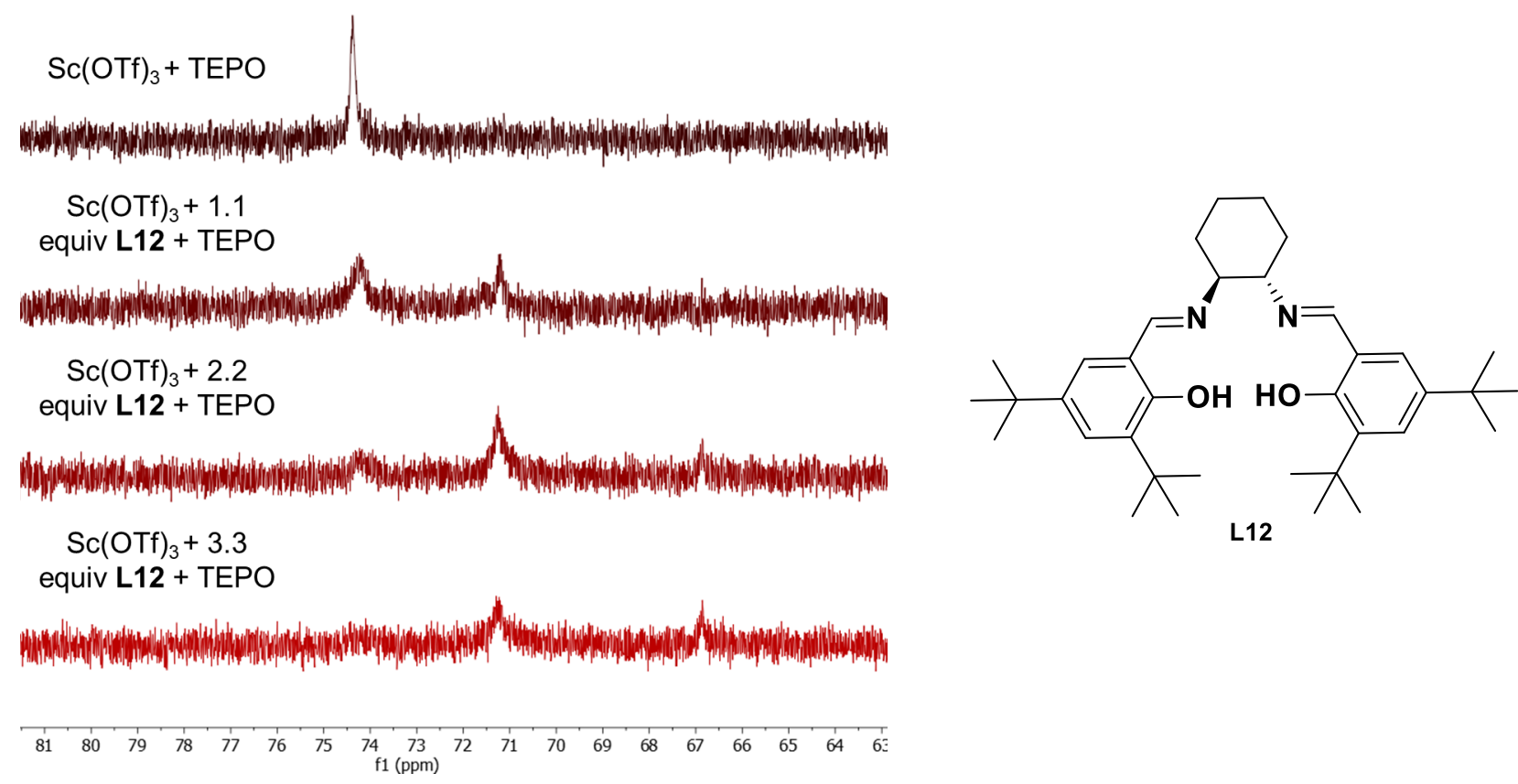

Figure S27. Effect of ligand stoichiometry on ${ }^{31} \mathrm{P}$ data for TEPO/Sc(OTf $)_{3} / \mathbf{L 1 2}$ mixtures 


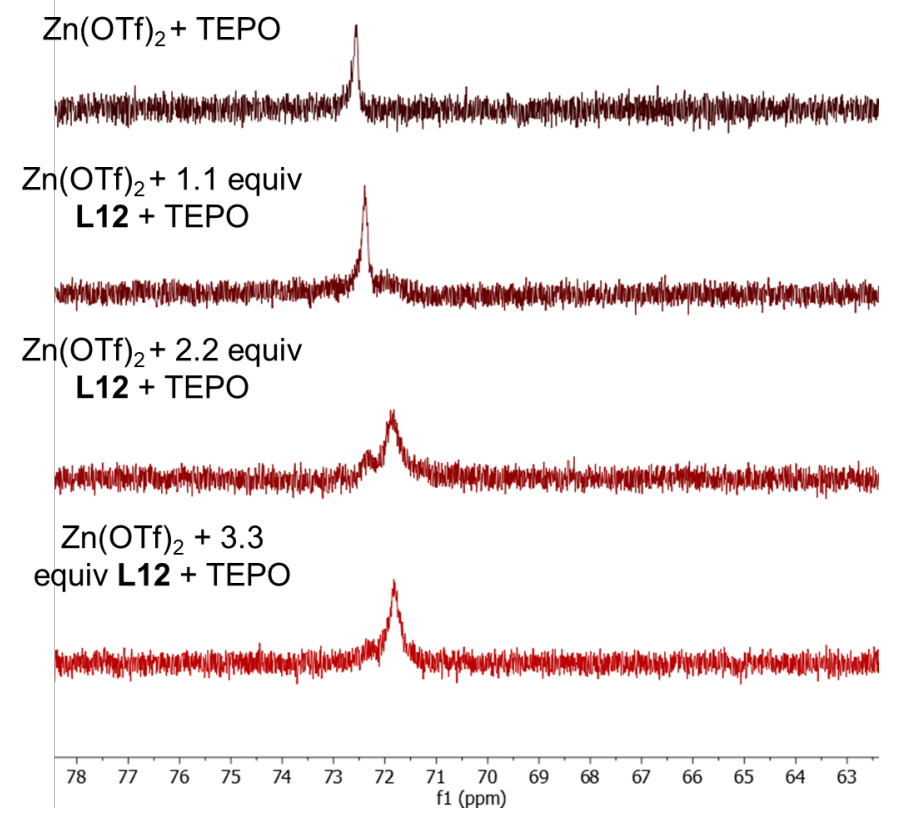

Figure S28. Effect of ligand stoichiometry on ${ }^{31} \mathrm{P}$ data for TEPO/Zn $(\mathrm{OTf})_{2} / \mathbf{L 1 2}$ mixtures

\section{Catalytic activity data}

To assess the ability of ${ }^{31} \mathrm{P}$ NMR binding data to predict catalytic activity, NMR yields for various catalysts were determined for two reactions (a Friedel-Crafts alkylation of indole and a Povarov annulation) and compared to TEPO and MBPO shift data.

Reaction yields were recorded at $3 \mathrm{~h}$ and $16 \mathrm{~h}$. The presence or absence of stirring during the reaction was observed to affect reaction rates due to the insolubility of the metal salts. Because of this, reaction rates could not be obtained using NMR spectroscopy or other methods where stirring was not possible. All reactions were stirred on the same stir plate with the same rate of stirring to control for this effect. 


\section{VIIa. Reaction Yields at $3 \mathrm{~h}$}

Table S11. Correlating yields at $3 \mathrm{~h}$ and $\Delta \delta\left({ }^{31} \mathrm{P}\right)$ data for the Povarov annulation ${ }^{\mathrm{a}}$

\begin{tabular}{cccccc}
\hline Lewis acid complex & $\begin{array}{c}\text { Annulation } \\
\text { yield }(\%)^{\mathrm{d}}\end{array}$ & $\mathrm{dr}$ & $\begin{array}{c}\text { TEPO } \\
\delta \Delta\left({ }^{31} \mathrm{P}\right) \\
(\mathrm{ppm})\end{array}$ & $\begin{array}{c}\text { TEMBP } \\
\delta \Delta\left({ }^{31} \mathrm{P}\right) \\
(\mathrm{ppm})\end{array}$ & $\begin{array}{c}\text { MBPO } \\
\delta \Delta\left({ }^{31} \mathrm{P}\right) \\
(\mathrm{ppm})\end{array}$ \\
\hline $\mathrm{Sc}(\mathrm{OTf})_{3} / \mathrm{AgSbF}_{6}$ & 86 & $49: 51$ & 44.1 & 2.7 & 18.7 \\
$\mathrm{Sc}(\mathrm{OTf})_{3} / \mathrm{NaBArF}$ & 45 & $44: 56$ & 27.2 & 2.7 & 17.3 \\
$\mathrm{Bi}(\mathrm{OTf})_{3}$ & 71 & $63: 37$ & 34.4 & 3.8 & 15.4 \\
$\mathrm{In}(\mathrm{OTf})_{3} / \mathrm{NaSbF}_{6}$ & 52 & $55: 45$ & 25.4 & 2.5 & 22.4 \\
$\mathrm{In}(\mathrm{OTf})_{3}$ & 19 & $61: 39$ & 25.3 & 2.7 & 22.8 \\
$\mathrm{Sc}(\mathrm{OTf})_{3}$ & 13 & $63: 37$ & 23.8 & 2.7 & 17.4 \\
$\mathrm{Sc}(\mathrm{OTf})_{3} / \mathrm{NaSbF}_{6}$ & 10 & $63: 37$ & 23.8 & 2.1 & 18.6 \\
$\mathrm{Sc}(\mathrm{OTf})_{3} / \mathbf{L} \mathbf{L} / \mathrm{AgSbF}_{6}$ & 11 & $36: 64$ & 24.1 & 2.6 & 17.2 \\
$\mathrm{Sc}(\mathrm{OTf})_{3} / \mathbf{L 1} / \mathrm{NaBArF}$ & 28 & $61: 39$ & 27.0 & 2.5 & 17.9 \\
$\mathrm{Ti}(\mathrm{OiPr})_{2}(\mathrm{OAr})_{2}{ }^{\mathrm{c}}$ & 0 & n.d. & 25.3 & 3.8 & 12.3 \\
$\mathrm{ZnCl}{ }^{{ }^{b}}$ & 18 & $61: 39$ & 19.3 & 1.3 & 7.2 \\
$\mathrm{Sc}(\mathrm{OTf})_{3} / \mathbf{L 1}$ & 0 & n.d. & 20.7 & 2.4 & 18.9 \\
$\mathrm{YCl}{ }_{3}{ }^{\mathrm{b}}$ & 0 & n.d. & 19.6 & 1.7 & 15.6 \\
$\mathrm{Y}(\mathrm{OTf})_{3}{ }^{\mathrm{b}}$ & 0 & n.d. & 17.3 & 1.9 & 10.6 \\
$\mathrm{La}(\mathrm{OTf})_{3}{ }^{{ }^{b}}$ & 0 & n.d. & 15.3 & 2.2 & 8.7
\end{tabular}

${ }^{\mathrm{a}} \Delta \delta\left({ }^{31} \mathrm{P}\right)$ data from Table $2(4.0 \mathrm{mM})$ unless otherwise indicated. $\mathbf{L 1}=2,6-\mathrm{Bis}[(3 \mathrm{a} R, 8 \mathrm{a} S)-(+)-8 H-$ indeno[1,2- $d]$ oxazolin-2-yl)pyridine ligand. ${ }^{\mathrm{b}} \Delta \delta\left({ }^{31} \mathrm{P}\right)$ data from Table $1(16.0 \mathrm{mM}) .{ }^{\mathrm{c}}(\mathrm{OAr})_{2}=(S)-(-)-$ $1,1^{\prime}-\mathrm{Bi}\left(2\right.$-naphthol). ${ }^{\mathrm{d}}$ Determined using ${ }^{1} \mathrm{H}$ NMR spectroscopy with internal standard. Allylation product was observed in $<5 \%$ for all catalysts.

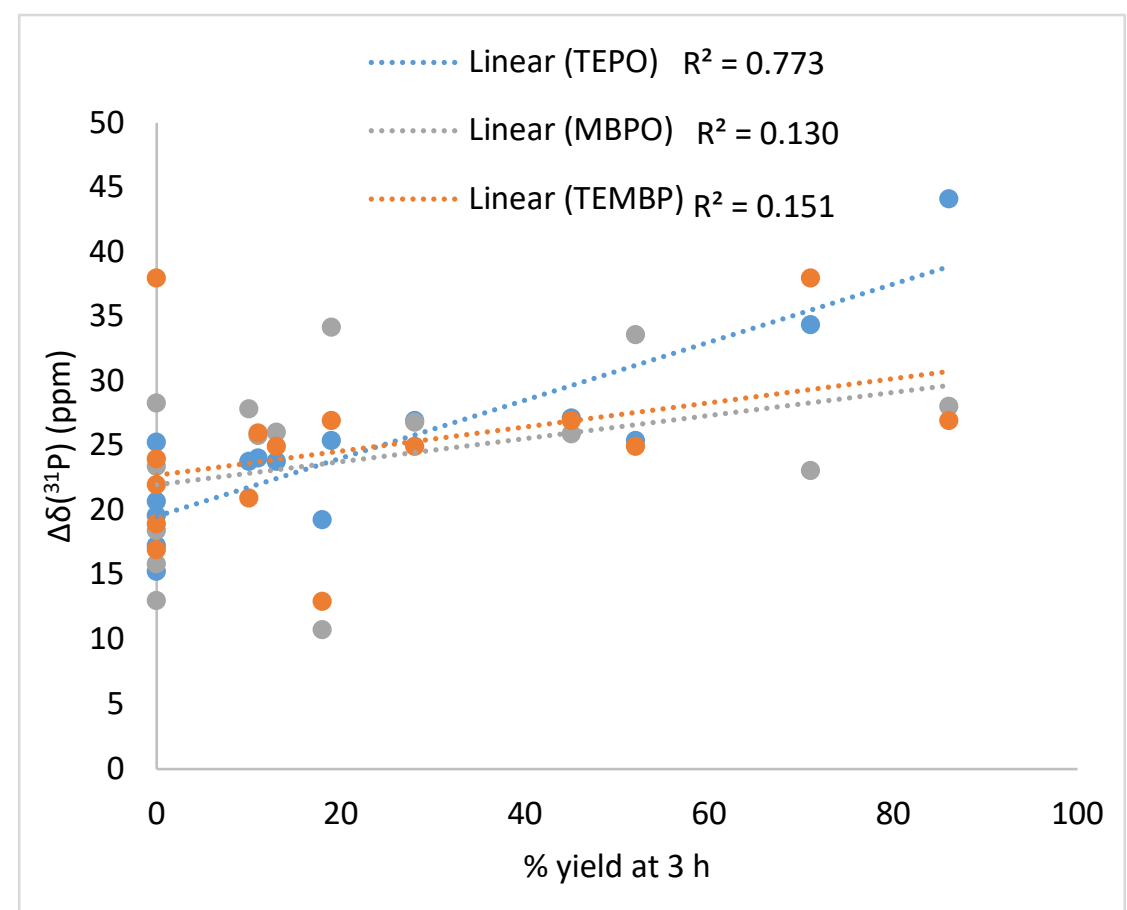


Figure S29. Correlation of reaction yields at $3 \mathrm{~h}$ and $\Delta \delta\left({ }^{31} \mathrm{P}\right)$ values for the Povarov annulation. TEMBP $\Delta \delta\left({ }^{31} \mathrm{P}\right)$ data was scaled by a factor of 10 and MBPO $\Delta \delta\left({ }^{31} \mathrm{P}\right)$ data was scaled by a factor of 1.5 so that correlation of shifts could be compared visually. This does not affect the $\mathrm{R}^{2}$ values.

Table S12. Correlating yields at $3 \mathrm{~h}$ and $\Delta \delta\left({ }^{31} \mathrm{P}\right)$ data for addition of indole to coumarin-3-carboxylates ${ }^{\mathrm{a}}$

\begin{tabular}{ccccc}
\hline $\begin{array}{c}\text { Lewis acid or Lewis } \\
\text { acid-ligand complex }\end{array}$ & $\begin{array}{c}\text { yield } \\
(\%)^{\mathrm{d}}\end{array}$ & $\begin{array}{c}\mathrm{MBPO} \\
\Delta \delta\left({ }^{3} \mathrm{P}\right) \\
(\mathrm{ppm})\end{array}$ & $\begin{array}{c}\text { TEPO } \\
\Delta \delta\left({ }^{31} \mathrm{P}\right) \\
(\mathrm{ppm})\end{array}$ & $\begin{array}{c}\text { TEMBP } \\
\Delta \delta\left({ }^{31} \mathrm{P}\right) \\
(\mathrm{ppm})\end{array}$ \\
\hline $\mathrm{Sc}(\mathrm{OTf})_{3} / \mathbf{L} \mathbf{L} / \mathrm{NaBArF}$ & 91 & 17.9 & 27 & 2.5 \\
$\mathrm{Y}(\mathrm{OTf})_{3} / \mathrm{NaBArF}$ & 80 & 16.6 & 24.6 & 2.0 \\
$\mathrm{Sc}(\mathrm{OTf})_{3} / \mathrm{NaBArF}$ & 83 & 17.3 & 27.2 & 2.7 \\
$\mathrm{YCl}_{3}{ }^{\mathrm{b}}$ & 83 & 15.6 & 19.6 & 1.7 \\
$\mathrm{Sc}(\mathrm{OTf})_{3}$ & 87 & 17.4 & 23.8 & 2.5 \\
$\mathrm{MgBr}_{2}{ }^{\mathrm{b}}$ & 31 & 10.0 & 12.3 & 1.5 \\
$\mathrm{Y}(\mathrm{OTf})_{3}{ }^{\mathrm{b}}$ & 56 & 10.6 & 17.3 & 1.9 \\
$\mathrm{Ti}(\mathrm{OiPr})_{2}(\mathrm{OAr})_{2}{ }^{\mathrm{c}}$ & 22 & 12.3 & 25.3 & 3.8 \\
$\mathrm{La}(\mathrm{OTf})_{3}{ }^{\mathrm{b}}$ & 0 & 8.7 & 15.3 & 2.2 \\
$\mathrm{ZnCl}{ }_{2}{ }^{\mathrm{b}}$ & 0 & 7.2 & 19.3 & 1.3 \\
$\mathrm{Ca}(\mathrm{OTf})_{2}$ & 0 & 7.3 & 8.0 & 2.4
\end{tabular}

${ }^{\mathrm{a}} \Delta \delta\left({ }^{31} \mathrm{P}\right)$ data from Table $2(4.0 \mathrm{mM})$ unless otherwise indicated. $\mathrm{L}=2,6-\mathrm{Bis}[(3 \mathrm{a} R, 8 \mathrm{a} S)-(+)-8 H-$ indeno[1,2- $d]$ oxazolin-2-yl)pyridine ligand. ${ }^{\mathrm{b}} \Delta \delta\left({ }^{31} \mathrm{P}\right)$ data from Table $1(16.0 \mathrm{mM}){ }^{\mathrm{c}}(\mathrm{OAr})_{2}=(S)-(-)-1,1^{\prime}-$ Bi(2-naphthol). ${ }^{\mathrm{d}}$ Determined using ${ }^{1} \mathrm{H}$ NMR spectroscopy with internal standard.

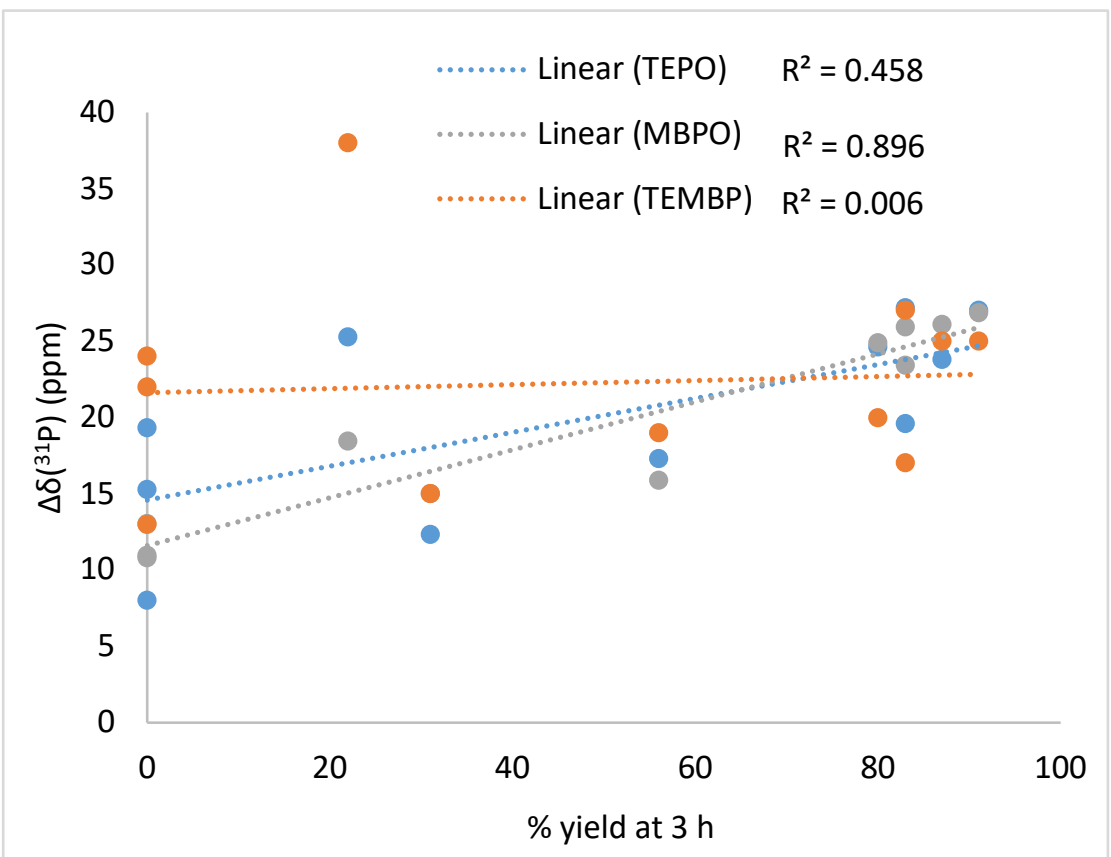

Figure S30. Correlation of catalytic activity and $\Delta \delta\left({ }^{31} \mathrm{P}\right)$ data for the addition of indole to coumarin-3carboxylates. TEMBP $\Delta \delta\left({ }^{31} \mathrm{P}\right)$ data was scaled by a factor of 10 and MBPO $\Delta \delta\left({ }^{31} \mathrm{P}\right)$ data was scaled by a factor of 1.5 so that correlation of shifts could be compared visually. This does not affect the $\mathrm{R}^{2}$ values. 
VIIb. Reaction Yields at $16 \mathrm{~h}$.

Table S13. Correlating yields at $16 \mathrm{~h}$ and $\Delta \delta\left({ }^{31} \mathrm{P}\right)$ data for the Povarov annulation ${ }^{\mathrm{a}}$

\begin{tabular}{|c|c|c|c|c|c|c|}
\hline Lewis acid complex & $\begin{array}{l}\text { Annulation } \\
\text { yield }(\%)^{\mathrm{d}}\end{array}$ & $\mathrm{dr}$ & $\begin{array}{c}\text { Allylation } \\
\text { yield } \\
(\%)^{\mathrm{d}} \\
\end{array}$ & $\begin{array}{c}\text { TEPO } \\
\delta \Delta\left({ }^{31} \mathrm{P}\right) \\
(\mathrm{ppm})\end{array}$ & $\begin{array}{c}\text { TEMBP } \\
\delta \Delta\left({ }^{31} \mathrm{P}\right) \\
(\mathrm{ppm})\end{array}$ & $\begin{array}{c}\mathrm{MBPO} \\
\delta \Delta\left({ }^{31} \mathrm{P}\right) \\
(\mathrm{ppm}) \\
\end{array}$ \\
\hline $\mathrm{Sc}(\mathrm{OTf})_{3} / \mathrm{AgSbF}_{6}$ & 100 & $46: 55$ & 3 & 44.1 & 2.7 & 18.7 \\
\hline $\mathrm{Sc}(\mathrm{OTf})_{3} / \mathrm{NaBArF}$ & 77 & $43: 57$ & 3 & 27.2 & 2.7 & 17.3 \\
\hline $\mathrm{Bi}(\mathrm{OTf})_{3}$ & 40 & $57: 43$ & 0 & 34.4 & 3.8 & 15.4 \\
\hline $\operatorname{In}(\mathrm{OTf})_{3} / \mathrm{NaSbF}_{6}$ & 38 & $42: 58$ & 0 & 25.4 & 2.5 & 22.4 \\
\hline $\operatorname{In}(\mathrm{OTf})_{3}$ & 36 & $61: 39$ & 0 & 25.3 & 2.7 & 22.8 \\
\hline $\mathrm{Sc}(\mathrm{OTf})_{3}$ & 32 & $64: 36$ & 4 & 23.8 & 2.7 & 17.4 \\
\hline $\mathrm{Sc}(\mathrm{OTf})_{3} / \mathrm{NaSbF}_{6}$ & 30 & $59: 41$ & 0 & 23.8 & 2.1 & 18.6 \\
\hline $\mathrm{Sc}(\mathrm{OTf})_{3} / \mathbf{L} \mathbf{1} / \mathrm{AgSbF}_{6}$ & 30 & $59: 41$ & 0 & 24.1 & 2.6 & 17.2 \\
\hline $\mathrm{Sc}(\mathrm{OTf})_{3} / \mathbf{L} \mathbf{1} / \mathrm{NaBArF}$ & 27 & $58: 42$ & 0 & 27.0 & 2.5 & 17.9 \\
\hline $\mathrm{ScCl}_{3}(\mathrm{THF})_{3}$ & 25 & $53: 47$ & 15 & 21.5 & 3.0 & 14.6 \\
\hline $\mathrm{Ti}(\mathrm{OiPr})_{2}(\mathrm{OAr})_{2}{ }^{\mathrm{c}}$ & 15 & $59: 41$ & 0 & 25.3 & 3.8 & 12.3 \\
\hline $\mathrm{ZnCl}_{2}{ }^{\mathrm{b}}$ & 7 & $43: 57$ & 0 & 19.3 & 1.3 & 7.2 \\
\hline $\mathrm{Sc}(\mathrm{OTf})_{3} / \mathbf{L} \mathbf{1}$ & 3 & $62: 38$ & 14 & 20.7 & 2.4 & 18.9 \\
\hline $\mathrm{YCl}_{3}^{\mathrm{b}}$ & 3 & $61: 39$ & 0 & 19.6 & 1.7 & 15.6 \\
\hline $\mathrm{Y}(\mathrm{OTf})_{3}{ }^{\mathrm{b}}$ & 2 & n.d. & 0 & 17.3 & 1.9 & 10.6 \\
\hline $\mathrm{La}(\mathrm{OTf})_{3}{ }^{\mathrm{b}}$ & 0 & n.d. & 0 & 15.3 & 2.2 & 8.7 \\
\hline
\end{tabular}

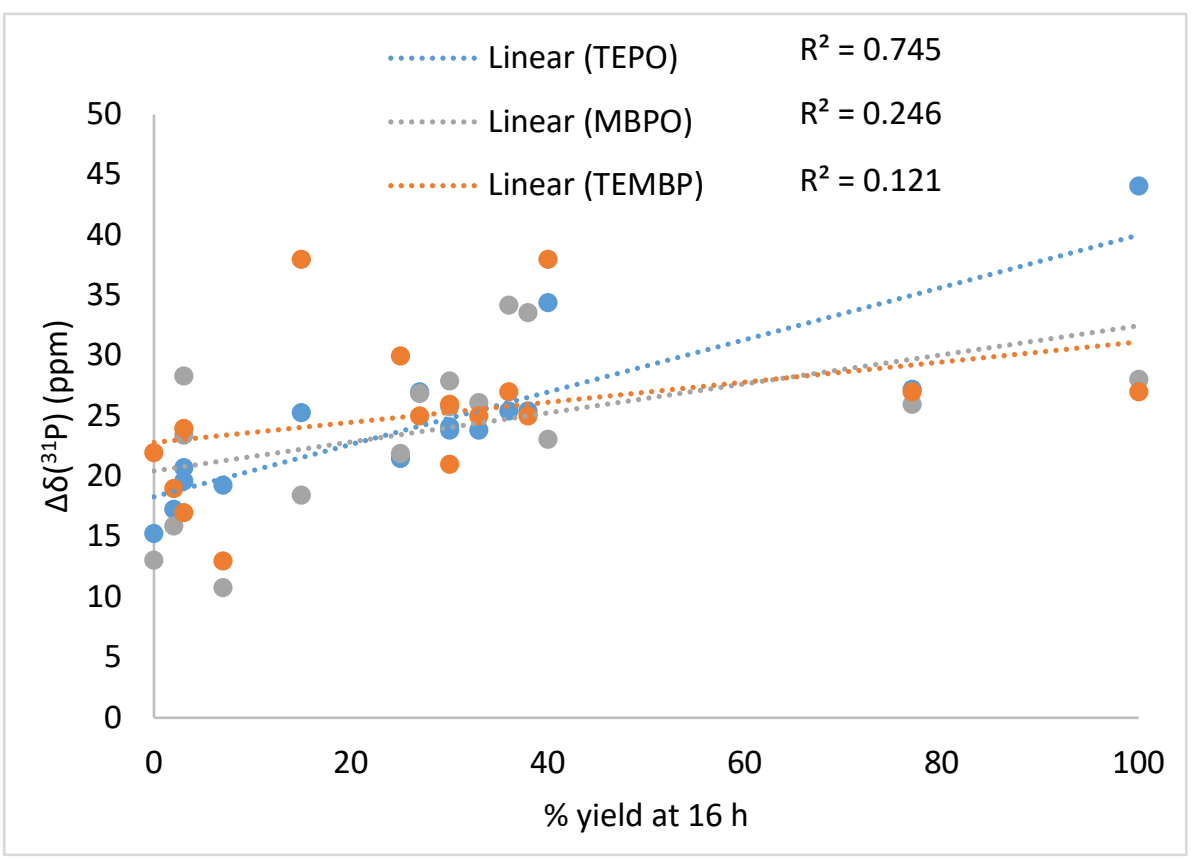


Figure S31. Correlation of reaction yields at $16 \mathrm{~h}$ and $\Delta \delta\left({ }^{31} \mathrm{P}\right)$ values for the Povarov annulation. TEMBP $\Delta \delta\left({ }^{31} \mathrm{P}\right)$ data was scaled by a factor of 10 and MBPO $\Delta \delta\left({ }^{31} \mathrm{P}\right)$ data was scaled by a factor of 1.5 so that correlation of shifts could be compared visually. This does not affect the $\mathrm{R}^{2}$ values.

Table S14. Correlating yields at $16 \mathrm{~h}$ and $\Delta \delta\left({ }^{31} \mathrm{P}\right)$ data for addition of indole to coumarin-3-carboxylates ${ }^{\mathrm{a}}$

\begin{tabular}{ccccc}
\hline $\begin{array}{c}\text { Lewis acid or Lewis } \\
\text { acid-ligand complex }\end{array}$ & $\begin{array}{c}\text { yield } \\
(\%)^{\mathrm{d}}\end{array}$ & $\begin{array}{c}\text { MBPO } \\
\Delta \delta\left({ }^{31} \mathrm{P}\right) \\
(\mathrm{ppm})\end{array}$ & $\begin{array}{c}\text { TEPO } \\
\Delta \delta\left({ }^{31} \mathrm{P}\right) \\
(\mathrm{ppm})\end{array}$ & $\begin{array}{c}\text { TEMBP } \\
\Delta \delta\left({ }^{31} \mathrm{P}\right) \\
(\mathrm{ppm})\end{array}$ \\
\hline $\mathrm{Sc}(\mathrm{OTf})_{3} / \mathbf{L 1} / \mathrm{NaBArF}$ & 92 & 17.9 & 27 & 2.5 \\
$\mathrm{Y}(\mathrm{OTf})_{3} / \mathrm{NaBArF}$ & 86 & 16.6 & 24.6 & 2.0 \\
$\mathrm{Sc}(\mathrm{OTf})_{3} / \mathrm{NaBArF}$ & 85 & 17.3 & 27.2 & 2.7 \\
$\mathrm{YCl}_{3}{ }^{\mathrm{b}}$ & 84 & 15.6 & 19.6 & 1.7 \\
$\mathrm{Sc}(\mathrm{OTf})_{3}$ & 79 & 17.4 & 23.8 & 2.5 \\
$\mathrm{MgBr}_{2}{ }^{\mathrm{b}}$ & 40 & 10.0 & 12.3 & 1.5 \\
$\mathrm{Y}(\mathrm{OTf})_{3}{ }^{\mathrm{b}}$ & 16 & 10.6 & 17.3 & 1.9 \\
$\mathrm{Ti}(\mathrm{OiPr})_{2}(\mathrm{OAr})_{2}{ }^{\mathrm{c}}$ & 13 & 12.3 & 25.3 & 3.8 \\
$\mathrm{La}(\mathrm{OTf})_{3}{ }^{\mathrm{b}}$ & 4 & 8.7 & 15.3 & 2.2 \\
$\mathrm{ZnCl}{ }^{\mathrm{b}}$ & 3 & 7.2 & 19.3 & 1.3 \\
$\mathrm{Ca}(\mathrm{OTf})_{2}$ & 0 & 7.3 & 8.0 & 2.4
\end{tabular}

${ }^{\mathrm{a}} \Delta \delta\left({ }^{31} \mathrm{P}\right)$ data from Table $2(4.0 \mathrm{mM})$ unless otherwise indicated. $\mathrm{L}=2,6-\mathrm{Bis}[(3 \mathrm{a} R, 8 \mathrm{a} S)-(+)-8 H-$ indeno[1,2- $d]$ oxazolin-2-yl)pyridine ligand. ${ }^{\mathrm{b}} \Delta \delta\left({ }^{31} \mathrm{P}\right)$ data from Table $1(16.0 \mathrm{mM}){ }^{\mathrm{c}}(\mathrm{OAr})_{2}=(S)-(-)-1,1^{\prime}-$ Bi(2-naphthol). ${ }^{\mathrm{d}}$ Determined using ${ }^{1} \mathrm{H}$ NMR spectroscopy with internal standard.

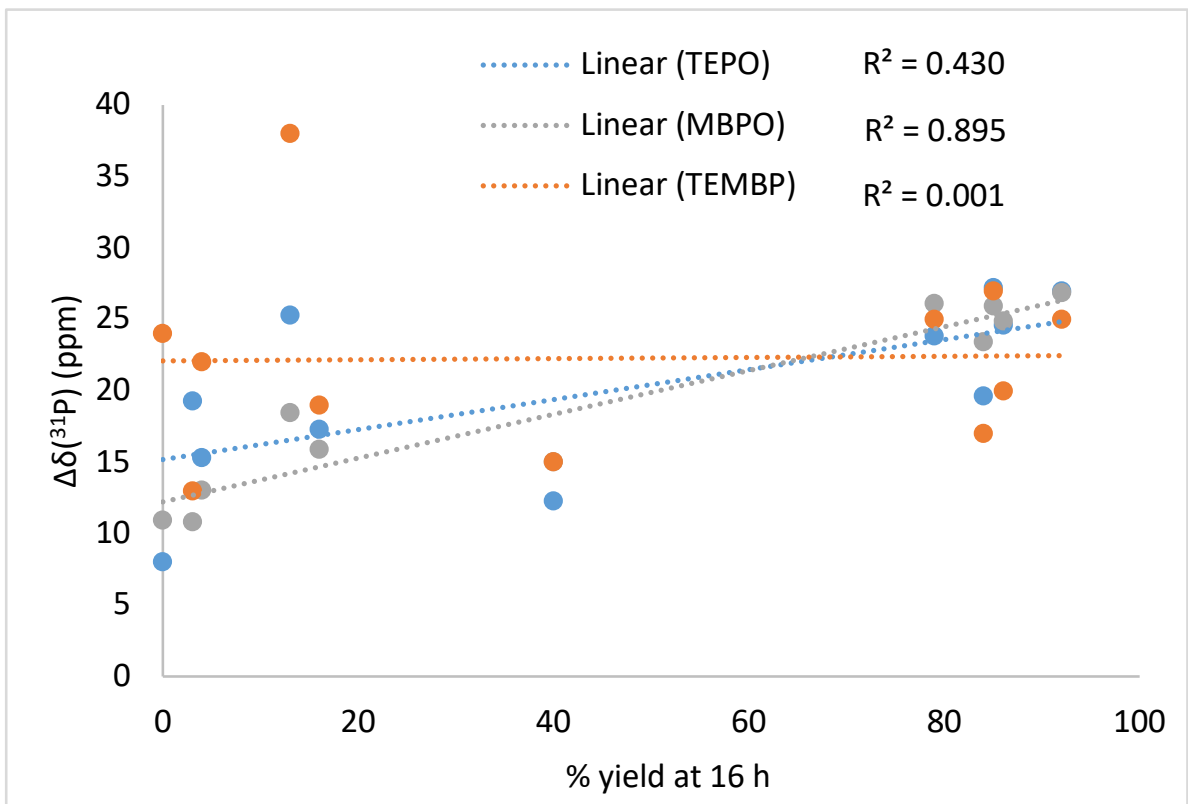

Figure S32. Correlation of catalytic activity and $\Delta \delta\left({ }^{31} \mathrm{P}\right)$ data for the addition of indole to coumarin-3carboxylates. TEMBP $\Delta \delta\left({ }^{31} \mathrm{P}\right)$ data was scaled by a factor of 10 and MBPO $\Delta \delta\left({ }^{31} \mathrm{P}\right)$ data was scaled by a factor of 1.5 so that correlation of shifts could be compared visually. This does not affect the $\mathrm{R}^{2}$ values. 


\section{Correlation of $\Delta \delta\left({ }^{31} \mathrm{P}\right)$, charge density and catalytic activity}

The correlation between charge density, $\Delta \delta\left({ }^{31} \mathrm{P}\right)$ and catalytic activity were evaluated. Charge density is defined as $\mathrm{z} / \mathrm{r}$ where $\mathrm{z}$ is charge and $\mathrm{r}$ is atomic radius. The overall correlation between TEPO binding $\Delta \delta\left({ }^{31} \mathrm{P}\right)$ values and charge density is low $\left(\mathrm{R}^{2}=0.16\right)$, as shown in Figure $\mathrm{S} 33$.

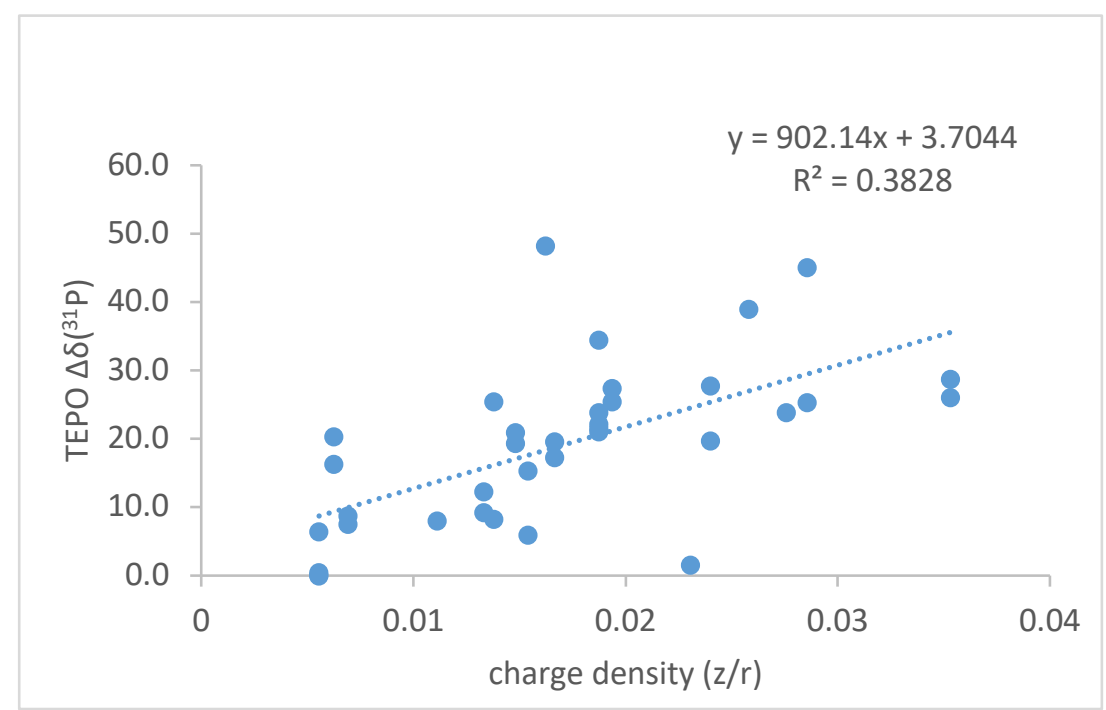

Figure S33. Correlation of charge density and TEPO binding $\Delta \delta\left({ }^{31} \mathrm{P}\right)$ values for all Lewis acids tested.

A significantly higher correlation between charge density and $\Delta \delta\left({ }^{31} \mathrm{P}\right)$ values is observed for Lewis acids used as catalysts in the Povarov annulation and indole addition to coumarin-3-carboxylates $\left(\mathrm{R}^{2}=0.75\right)$ as shown in Figure S34. 


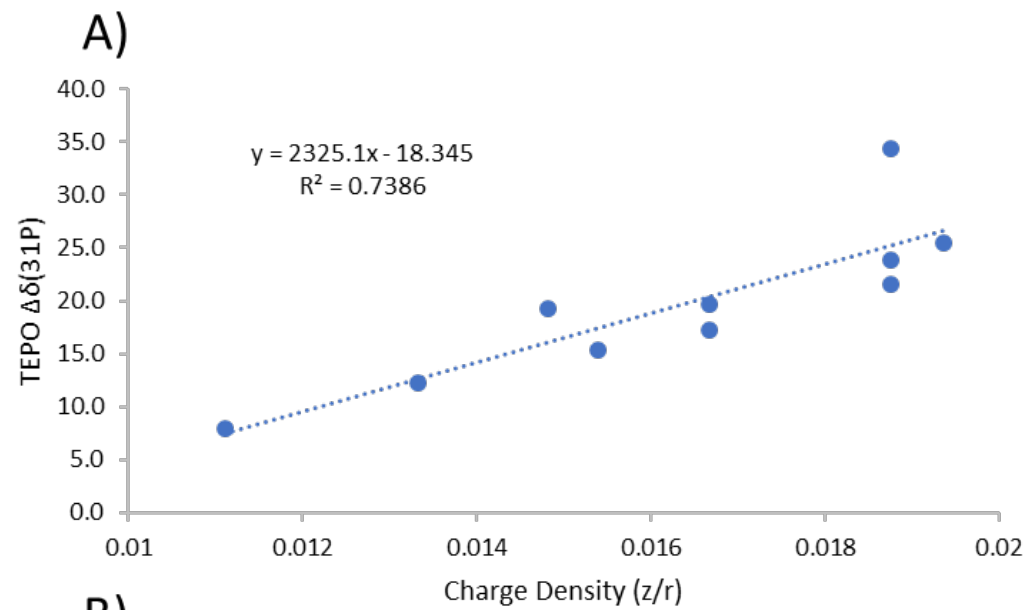

B)

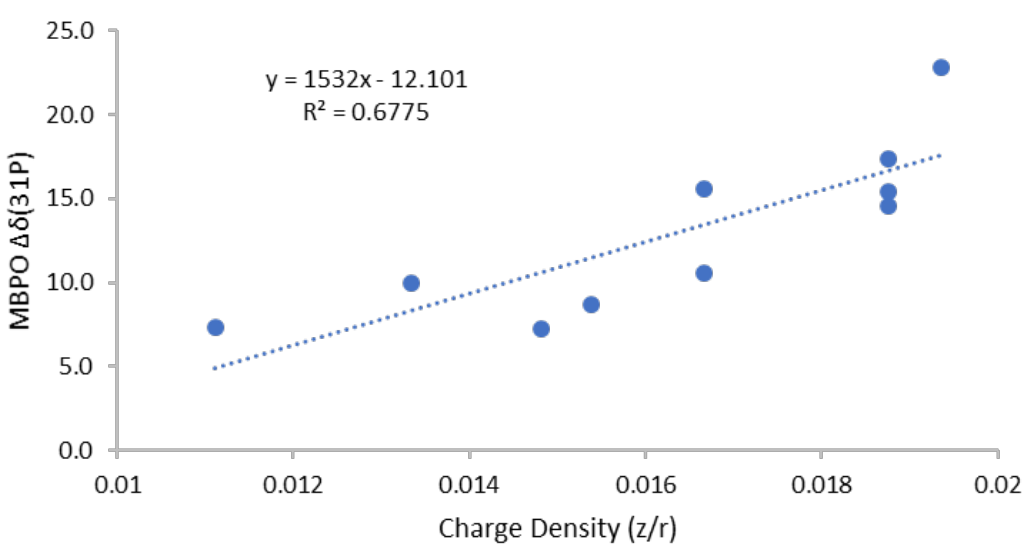

Figure S34. Correlation of charge density and A) TEPO or B) MBPO binding $\Delta \delta\left({ }^{31} \mathrm{P}\right)$ values for Lewis acids shown in Table S16. ${ }^{a}$ 
Table S15. Catalytic activity, charge density $(\mathrm{z} / \mathrm{r})$ and $\Delta \delta\left({ }^{31} \mathrm{P}\right)$ data for Povarov annulation and addition of indole to coumarin-3-carboxylates ${ }^{\mathrm{a}}$

\begin{tabular}{cccccc}
\hline Lewis acid & $\begin{array}{c}\text { TEPO } \\
\Delta \delta\left({ }^{31} \mathrm{P}\right) \\
(\mathrm{ppm})\end{array}$ & $\begin{array}{c}\text { MBPO } \\
\Delta \delta\left({ }^{31} \mathrm{P}\right) \\
(\mathrm{ppm})\end{array}$ & $\begin{array}{c}\text { charge density } \\
(\mathrm{z} / \mathrm{r})\end{array}$ & $\begin{array}{c}\text { Indole addition } \\
\text { to coumarin } \\
\text { yield at } 16 \mathrm{~h} \\
(\%)\end{array}$ & $\begin{array}{c}\text { Povarov } \\
\text { annulation yield } \\
\text { at 16 } \mathrm{h}(\%)\end{array}$ \\
\hline $\mathrm{Bi}(\mathrm{OTf})_{3}$ & 34.4 & 15.4 & 0.01875 & $-\mathrm{b}^{\mathrm{b}}$ & 40 \\
$\mathrm{In}(\mathrm{OTf})_{3}$ & 25.3 & 22.8 & 0.01935 & $-\mathrm{b}$ & 36 \\
$\mathrm{Sc}(\mathrm{OTf})_{3}$ & 23.8 & 17.4 & 0.01875 & 79 & 32 \\
$\mathrm{ScCl}_{3}(\mathrm{THF})_{3}$ & 21.5 & 14.6 & 0.01875 & $-\mathrm{b}$ & 25 \\
$\mathrm{ZnCl}_{2}$ & 19.3 & 7.2 & 0.01481 & 3 & 7 \\
$\mathrm{YCl}_{3}$ & 19.6 & 15.6 & 0.01666 & 84 & 3 \\
$\mathrm{Y}(\mathrm{OTf})_{3}$ & 17.3 & 10.6 & 0.01666 & 16 & 2 \\
$\mathrm{La}(\mathrm{OTf})_{3}$ & 15.3 & 8.7 & 0.01538 & 4 & 0 \\
$\mathrm{MgBr}_{2}$ & 12.3 & 10.0 & 0.01333 & 40 & $-\mathrm{b}$ \\
$\mathrm{Ca}(\mathrm{OTf})_{2}$ & 8.0 & 7.3 & 0.01111 & 0 & $-\mathrm{b}$
\end{tabular}

${ }^{a}$ Charge density $(\mathrm{z} / \mathrm{r})$ was calculated based on $\frac{z}{r}=$ charge density, where $\mathrm{z}=$ charge and $\mathrm{r}=$ atomic radius. For example, for indium: $\frac{3}{155}=0.0193355$. ${ }^{b}$ Not tested for the indicated reaction.

The correlation between charge density, TEPO $\Delta \delta\left({ }^{31} \mathrm{P}\right)$ data and reaction yield in the Povarov annulation is shown in Figure S35. Charge density had a lower correlation with reaction yields $\left(\mathrm{R}^{2}=0.78\right)$ than with TEPO binding $\Delta \delta\left({ }^{31} \mathrm{P}\right)$ values $\left(\mathrm{R}^{2}=0.81\right)$. The correlation between charge density and reaction yield is lower for the addition of indole to coumarin-3-carboxylates $\left(\mathrm{R}^{2}=0.40\right)$ (Figure S36). The correlation between TEPO $\Delta \delta\left({ }^{31} \mathrm{P}\right)$ data and reaction yield is also low for this reaction $\left(\mathrm{R}^{2}=0.37\right)$ (Figure $\left.\mathrm{S} 36 \mathrm{~B}\right)$. The addition of indole to coumarin-3-carboxylates proceeds via chelating activation of the coumarin-3carboxlate by the Lewis acid. This activation mode is better modeled by the chelating MBPO probe than the non-chelating TEPO probe. The correlation between MBPO $\Delta \delta\left({ }^{31} \mathrm{P}\right)$ values and reaction yield is 0.91 (Figure S36A), indicating that MBPO better models catalytic activity for reactions that require bidentate binding of the electrophile. 


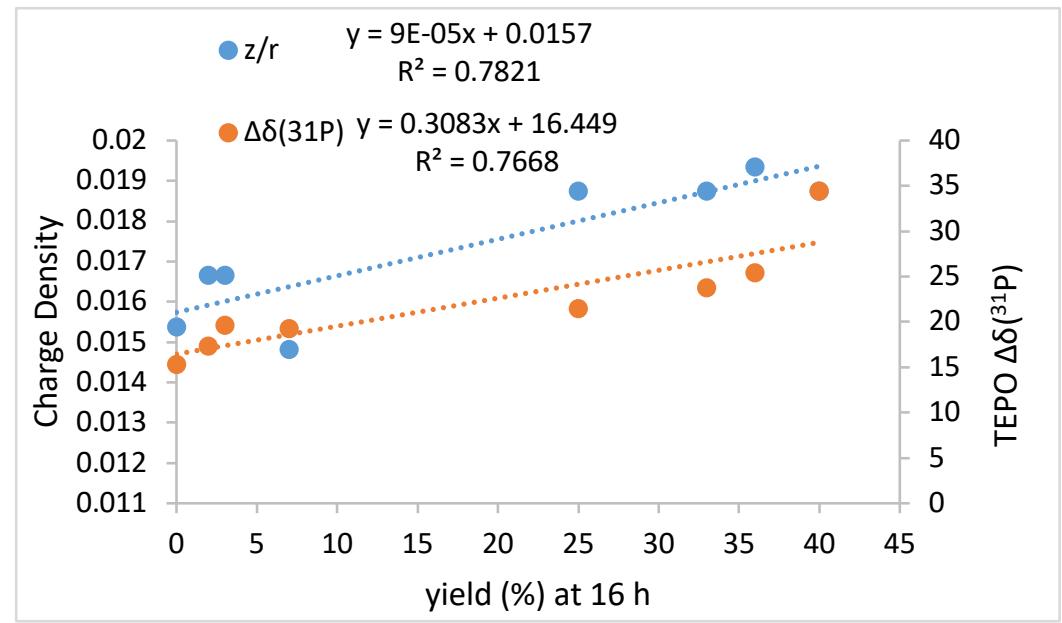

Figure S35. Correlation of charge density, TEPO $\Delta \delta\left({ }^{31} \mathrm{P}\right)$ data and reaction yield at $16 \mathrm{~h}$ for the Povarov annulation.
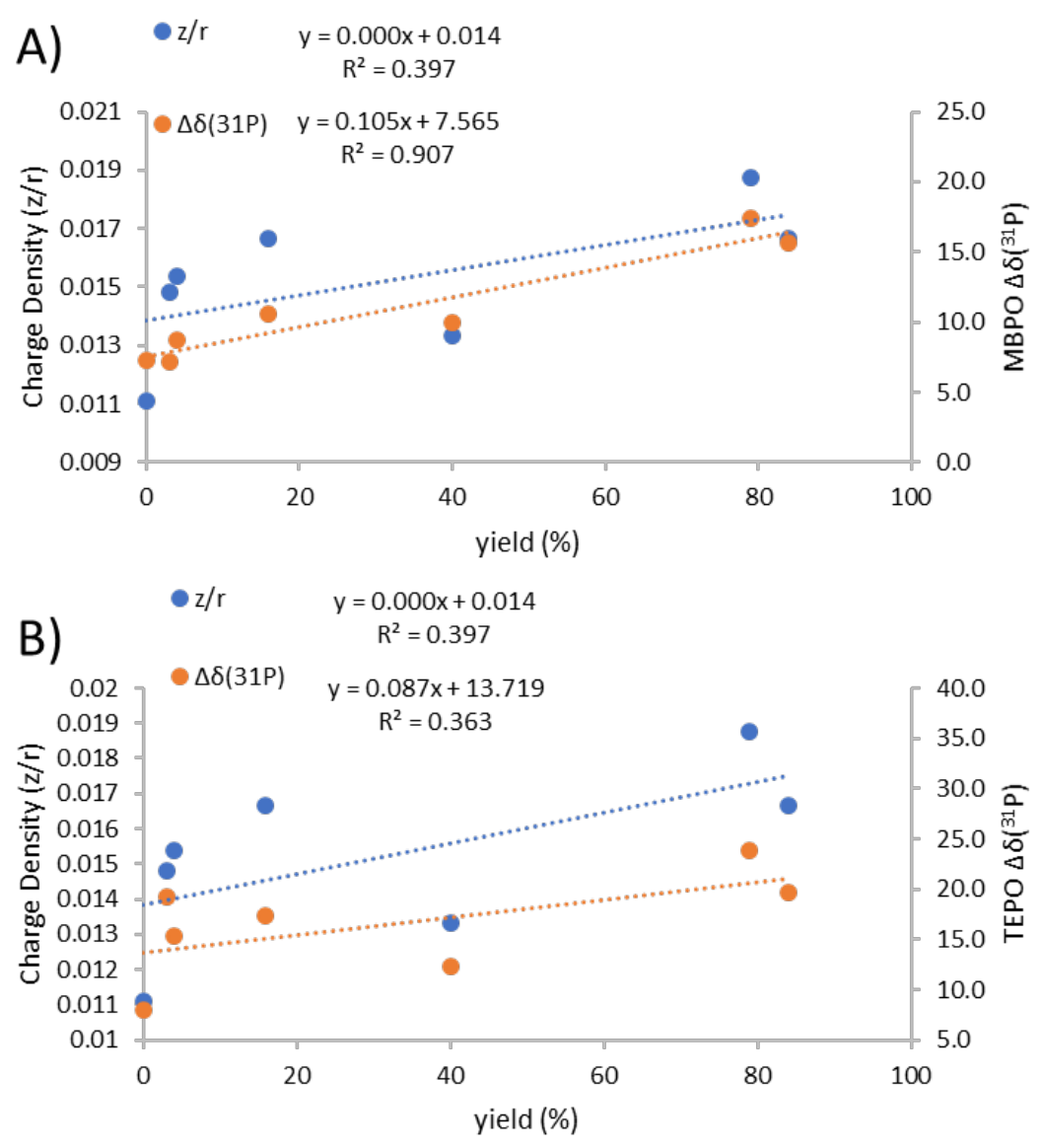

Figure S36. A) Correlation of charge density, MBPO $\Delta \delta\left({ }^{31} \mathrm{P}\right)$ data and reaction yield at $16 \mathrm{~h}$ for the indole addition to coumarin-3-carboxylates. B) Correlation of charge density, TEPO $\Delta \delta\left({ }^{31} \mathrm{P}\right)$ data and reaction yield for the indole addition to coumarin-3-carboxylates. 